\title{
Annotationes Epigraphicae VII \\ Zu einigen Inschriften aus den römischen Provinzen Germania inferior und Germania superior*
}

Andreas Kakoschke

I.

Im Jahre 1976 fand sich in Köln-Deutz/CCAA, im Bereich des spätantiken Kastells, ein in mehrere Teile zerbrochener unvollständig erhaltener Weihealtar (Höhe $58 \mathrm{~cm}-$ Breite $40 \mathrm{~cm}$ - Tiefe $12 \mathrm{~cm}$ ) aus Kalkstein (Abb.1). Die nicht ganz erhaltene Inschrift des Steins aus dem Jahr 252 n. Chr. gaben die Ersteditoren B. und H. GALSTERER in Umschrift wie folgt wieder: ${ }^{1}$

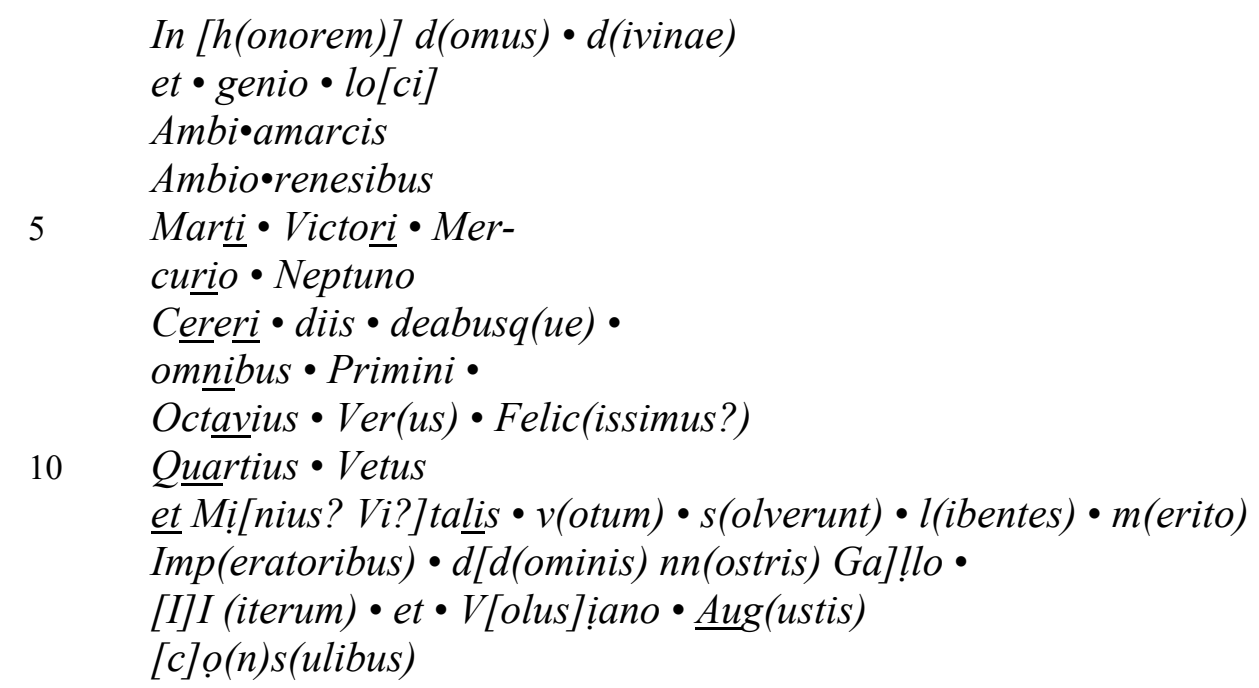

Diese Lesung der Sammelweihung wurde von B. und H. GALSTERER in einer späteren Publikation leicht korrigiert. So lesen die Autoren in Zeile 9 nun Felic(ior?, issimus), in Zeile 11 Mi[nicius? Vi?] talis und am Beginn von Zeile 13 nicht mehr [I]I (iterum), sondern nur noch et V[olus]iano. ${ }^{2}$

\footnotetext{
* Für die Bereitstellung einiger Photos danke ich Herrn Prof. Dr. Rainer Wiegels und Herrn Prof. Dr. Krešimir Matijević (CIL XIII/2-Projekt, Universität Flensburg/Trier) sowie Herrn Dr. Martin Kemkes (Landesmuseum Stuttgart) und Herrn Dr. Ortolf Harl (Universität Wien). - Ligaturen bzw. Nexus werden im Folgenden durch unterstrichene Buchstaben wiedergegeben. - Die Annotationes Epigraphicae I und II erschienen in der FeRA 26 und 27 (2015), 10-26 bzw. 20-42. Die Annotationes Epigraphicae III erschienen im GFA 19 (2015), 179-197, die Annotationes Epigraphicae IV in der FeRA 29 (2016), 43-76, die Annotationes Epigraphicae V in der FeRA 30 (2016), 1-25 und die Annotationes Epigraphicae VI in der FeRA 31 (2016), 1-24.

${ }^{1}$ GAlsterer 1981, 228-232. Nr.3 (mit Photo) = AE 1981, 660. S. ferner M. CARROLL-SPILleCKe, KJb 26 (1993), 441. Nr.XIV 38, BuRNs 1994, 180, EDH-Nr.HD005413 (jeweils mit identischer Lesung). Ein Photo der Inschrift (ohne Lesungen) veröffentlichte zuvor bereits H. BORGER u.a., Colonia antiqua, Brüssel 1977, 102-103. Nr.37. - Der Weihestein befindet sich heute im Römisch-Germanischen Museum in Köln (Inv.-Nr.76, 472.1).

${ }^{2}$ IKöln² 30-32. Nr.3 (mit Photo). S. auch ThomAs 2004, 133. Nr.6, EDCS Nr.01100011 (jeweils mit entsprechender Lesung). Nach Ga]llo (Zeile 12) fehlt in den IKöln² in der Transkription nun jedoch der Zeilentrenner.
} 
Die Lesung der Inschrift lässt sich darüber hinaus m.E. in weiteren Punkten verbessern: In Zeile 10 lautet die Lesung bei B. und H. GALSTERER Quartius - Vetus. In dem ausgebrochenen Raum zwischen dem Worttrenner und VETVS verbleibt jedoch eindeutig Platz für einen weiteren relativ breiten Buchstaben (Abb.2). Angesichts der Buchstabenabstände und des bekannten Namenmaterials kann es sich bei diesem Buchstaben nur um ein Q handeln. Möglicherweise ist von dem Q links unterhalb des V (von VETVS) auch noch der Rest des fein auslaufenden Schweifs zu erkennen. Der Dedikantenname in Zeile 10 lautete also Quartius [Q]uetus. Mit Quetus liegt eine oftmals verwendete Variante des lateinischen Cognomens Quietus vor. Im Gegensatz $\mathrm{zu}$ dem in Niedergermanien bisher unbekannten Vetus ist Quetus mehrfach in der Germania inferior bezeugt. Der Name fungierte auch als Basis für die hier belegten Pseudogentilnomina Quetius und Quetinius. ${ }^{3}$

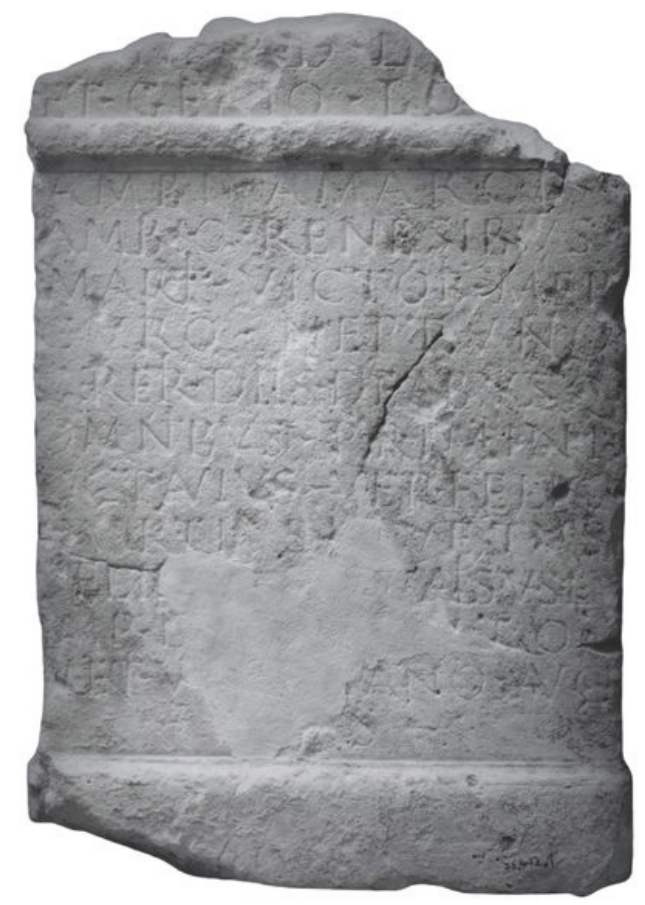

Abb.1: Weihinschrift aus Köln-Deutz/CCAA: AE 1981, 660 (C) Römisch-Germanisches Museum, Köln)

Für die Namen in Zeile 8 und 9 schlagen B. und H. GALSTERER die Lesung Primini( $i$ ) Octavius, Ver(us), Felic(ior? issimus?) vor. Die Autoren gehen also von einem vorangestellten Pseudogentiliz im Plural aus und sehen in den genannten Personen drei Mitglieder aus der Familie der Priminii. Obwohl man in diesem Fall eher, wenngleich nicht zwingend, ein I-longa am Ende von Primini erwartet hätte, ${ }^{4}$ hat diese Auf-

\footnotetext{
${ }^{3}$ Zu Vetus s. KaKoschKe 2008, 449. CN 3308. - Zu Quietus bzw. Quetus s. KaKoschKe 2008, $243-$ 244. CN 2520. Quetus findet sich in folgenden Inschriften aus Niedergermanien: CIL XIII $3613=\mathrm{AE}$ 2002, 1013 (Theux), 7943 [Iversheim (jedoch fraglich)], 8573 = LEHNER 1918, 155. Nr.339 (OssumBösinghoven, bei Haus Gripswald), AE 1990, $728=\mathrm{IKöln}^{2}$ 106-108. Nr.100 (Köln/CCAA). - Zu den Namen Quetius und Quetinius s. KAKOschKe 2014, 87-88, A. KAKOschKe, GFA 18 (2015), 189-192. Quetinius ist zweimal in Köln/CCAA bezeugt: CIL XIII $8344=$ IKöln $^{2}$ 368-369. Nr.442, AE 1981, 670 $=$ IKöln ${ }^{2}$ 64-66. Nr.44.

${ }^{4}$ Ein I-longa am Ende eines Gentilnomens im Nominativ Plural bieten z.B. folgende Inschriften aus den zwei germanischen Provinzen: CIL XIII 6097 (Speyer/Noviomagus; Claudi Mascellio et Clemens), 7505 (Bingen/Bingium; Privati Secundinus et Tertinus et Confinis), 8591 (Moers-Asberg/Asciburgium;
} 
fassung durchaus ihre Berechtigung. ${ }^{5}$ Allerdings lassen sich ebenso ohne weiteres zwei Namen lesen: Primini(us) Octavius und Ver(inius?) Felic(io?). Für diese Auffassung mag die Tatsache sprechen, dass ein lediglich mit VER angegebenes Gentiliz, bei dem es sich wohl um den (in Köln/CCAA gängigen/bekannten) Namen Verinius oder Veranius handelt, drei- bzw. viermal in der Stadt am Rhein bezeugt ist. ${ }^{6}$ Dagegen kann für die abgekürzte Form $\operatorname{Ver}(u s)$ aus den zwei germanischen Provinzen nur ein Beleg aus Mainz/Mogontiacum angeführt werden, ${ }^{7}$ obwohl das Cognomen gerade in der Germania inferior stark verbreitet war. ${ }^{8}$ Hinsichtlich der Lesung Primini(us) Octavius lässt sich anführen, dass die abgekürzte Form Primini (Wortstamm + i) für den Nominativ Singular Primini(us) durch weitere vergleichbare Belege gestützt wird. Der Form Primini(us) ist also keinesfalls außergewöhnlich. ${ }^{9}$ Ob zudem der etwas größere Abstand zwischen OCTAVIVS und VER FELIC in Zeile 9 für zwei Namen bzw. Personen spricht, sei dahingestellt.

Die Ergänzung des Namens in Zeile 11 ist - wie es B. und H. GALSTERER in der Umschrift durch Fragezeichen kenntlich machen - natürlich sehr spekulativ. Zudem sollte man berücksichtigen, dass es sich bei dem vermeinlichen I am Beginn der Lücke ebenso um ein E handeln kann. Es kommt also ferner eine Ergänzung zu mit ME beginnenden Gentilnomina, wie Memmius, Mettius oder Messius, in Frage.

Schließlich sei für die ersten zwei Zeilen der Inschrift die Lesung [I]n [h(onorem)] d(omus) d(ivinae) [I(ovi) O(ptimo) M(aximo) oder Iovi] / et genio lo[ci et] vorgeschlagen. Hierfür spricht die zu vermutende Verteilung der Buchstaben in den Anfangszeilen (Abb.2) und die Tatsache, dass der Genius loci (in Niedergermanien) fast immer mit und dann nach Iupiter Optimus Maximus genannt wird. Die Wortfolge in honorem domus divinae et genio loci ist auf jeden Fall ungewöhnlich. ${ }^{10}$

Nebenbei kann als Vergleich zu der Kölner Inschrift noch auf eine, auch von B. und H. GALSTERER erwähnte, Weihinschrift von vier Legionären der legio XXX Ulpia victrix aus Remagen/Rigomagus verwiesen werden, die nach Iupiter Optimus Maximus und dem Genius loci Mars, Herkules, Merkur und die Ambiomarcae nennt. $^{11}$

Die Kölner Weihinschrift kann demzufolge wie folgt gelesen werden:

M. T. Caesi M. f. Kaesones), 11810 (Mainz/Mogonticaum; Victori Ursus et Lupus), LUPA Nr.25504 (Mosbach-Diedesheim; Festi Festinus et Florianus).

${ }^{5}$ Eine vergleichbare Namensauflistung bietet z.B. die Weihinschrift CIL XIII 6102 (Speyer/Noviomagus; Flavi Ubtio Avitus Maximus) oder die Grabinschrift CIL XIII 7298 (Mainz-Kastel/Castellum Mattiacorum; Servandi Eternus Servanda Severina Barbara). S. ferner CIL XIII 1901 (Lyon/Lugdunum; Verini Ursa Aeternus Marinus Victor). In allen genannten Fällen wurde das I am Ende des Gentilnomens als I-longa eingeschlagen.

${ }^{6}$ AE 1974, 462 = IKöln² 422-423. Nr.537 (mit Photo) [P. Ver(---) Mascellio und P. Ver(---) Vitalis], 3. N. 198. Nr.206 = IKöln² 33. Nr.4 (mit Photo) [T. Ver(---) Sene(cio?)], IKöln² 155. Nr.166 (mit Photo) [M. Ver(---) Er[---]]. S. ferner einen Beleg aus Dieburg in Obergermanien: AE 2003, 1272 [Ver(---) Verrinus!]. - Zu Verinius s. KAKOSChKe 2006, 426-427. CN 1397. Zu Veranius s. KaKoschKe 2006, 423-424. CN 1389.

${ }^{7}$ CIL XIII 6801 [Volus(ius) Ver(us)].

${ }^{8}$ Zum Namen s. KAKOSCHKE 2008, 445-446. CN 3295.

${ }^{9}$ Verwiesen sei z.B. auf CIL XIII 6685 (Mainz/Mogontiacum; Secundi Mansuetus), 8003a (BonnEndenich/Bonna; Attici Maternus), AE 1993, 1223 [Butzbach; Accepti Sever(i)nus], 2011, 827 (Medard; Amandi Amandina), 1. N. 205. Nr.345 (Bad Cannstatt; Sereni Atticus) , 4. N. 474. Nr.30 (Stettfeld; Florentini Quintianus), K. WoElCKE, Germania 21 (1937), 131 (Frankfurt-Heddernheim/Nida; Mettini Messia). Zu Näherem s. A. KAKOsChKE, FeRA 26 (2015), 14-17.

${ }^{10} \mathrm{Im}$ gesamten epigraphischen Material findet sich nur ein weiterer Beleg für diese Wortfolge: AE 2011, $776=$ J. KRIER, Gallia 68/2 (2011), 322. Abb.10 (Photo) (Dalheim/Ricciacum).

${ }^{11}$ CIL XIII 7789 = LEHNER 1918, 46. Nr.99. 

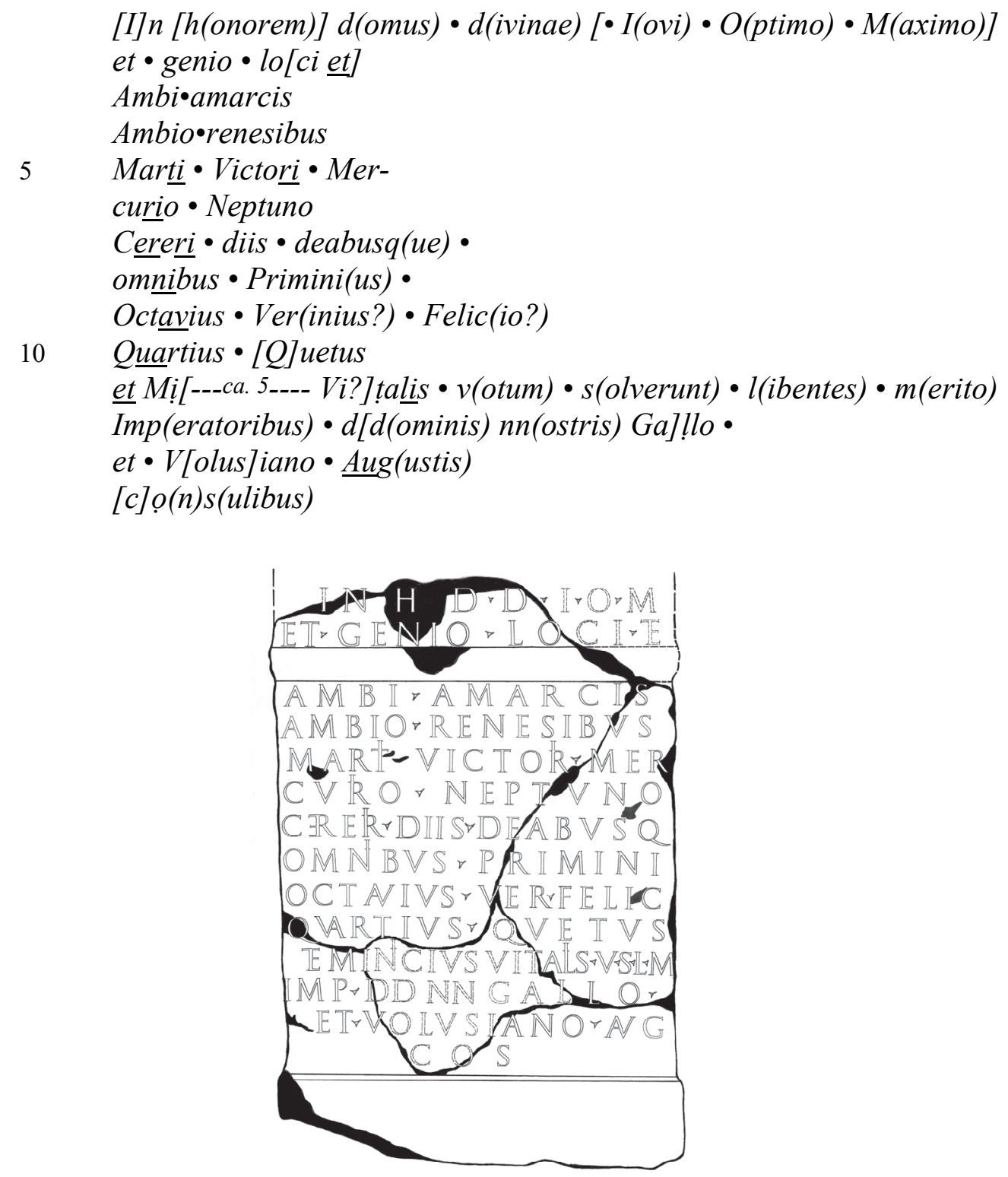

Abb.2: Leicht idealisierte Rekonstruktionszeichnung der Weihinschrift AE 1981, 660 aus Köln-Deutz/CCAA (Zeichnung A.K.)

II.

Ebenfalls aus Köln/CCAA stammt ein Weihestein (Höhe $57 \mathrm{~cm}$ - Breite $37 \mathrm{~cm}$ - Tiefe $17 \mathrm{~cm}$ ) aus Kalkstein (Abb.3) mit kaum noch zu lesender Inschrift. Das Stück fand sich im Jahre 1926 bei der Anlage einer neuen Gruftanlage für die Domgeistlichkeit vor dem Ostchor des Kölner Domes. Da der kleine Altar wohl lange als Treppenstufe diente, ist die fünfzeilige Inschrift nur noch schlecht zu entziffern. Dies gilt insbesondere für die zweite Zeile, in der der Name des Dedikanten stand. FREMERSDORF, der Ersteditor der Inschrift, gab den noch zu erkennenden Text in Majuskelschrift wie folgt wieder: ${ }^{12}$

\footnotetext{
${ }^{12}$ F. Fremersdorf, Germania 13 (1929), 132. Nr.1 = 1. N. 210-211. Nr.362. - Der Stein befindet sich heute im Römisch-Germanischen Museum in Köln (Inv.-Nr.26, 396).
} 


$\begin{array}{ll} & \text { LVCRETI[S] } \\ & \text { A I I A I I } \\ & \text { PRO SE } \\ & \text { ET SVIS } \\ & \text { VSL } M\end{array}$

Diesen Vorgaben entsprechend wurde die Inschrift aus dem zweiten Jahrhundert $\mathrm{n}$. Chr. nach der Auffindung - der genaue Zeitpunkt ist unbekannt - mit roter Farbe nachgezeichnet. ${ }^{13}$

Gleicht man die in der zweiten Zeile erhaltenen Hastenreste mit dem (bisher) bekannten Namenmaterial ab, zeigt sich, dass nur zu einem Namen ergänzt werden kann - zu ANAILLVS bzw. ANAILLA (Abb.4). Der keltische Name ist zweimal in der Gallia Begica bezeugt: in Plombières-les-Bains (dép. Vosges) im Gebiet der Leuker ${ }^{14}$ und in Montenach (dép. Moselle) im Gebiet der Mediomatriker. ${ }^{15}$ Ein Beleg in der Form Ana $\bullet$ iilus stammt aus Limoges/Augustoritum Lemovicum in Aquitanien. ${ }^{16}$ Zudem führte den Namen ein Töpfer aus dem aquitanischen Lezoux. ${ }^{17}$ Hinzu kommt schließlich ein Beleg für das aus dem Namen gebildete Pseudogentiliz Anaillius aus Bonn/Bonna. ${ }^{18}$

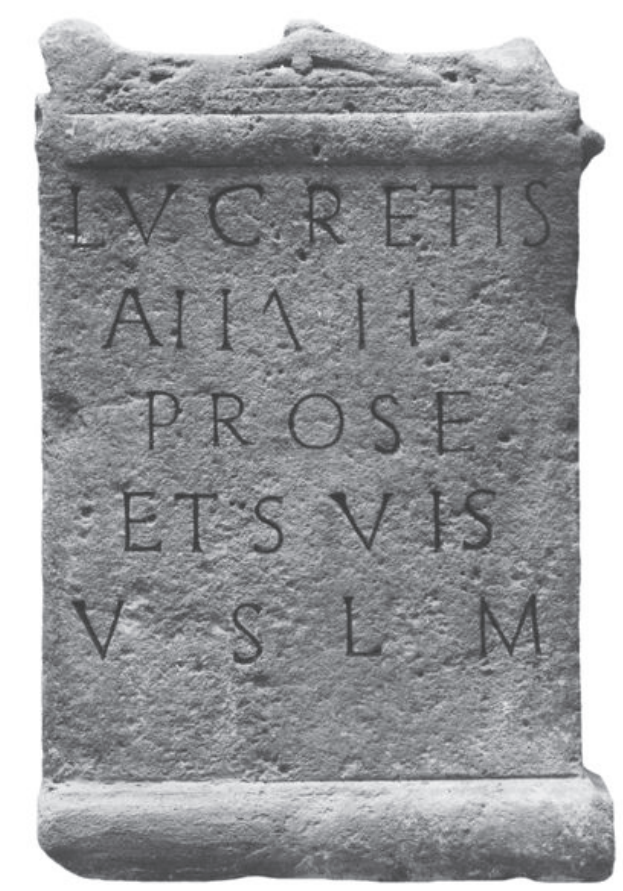

Abb.3: Weihinschrift aus Köln/CCAA: 1.N. 210-211. Nr.362

(C) Römisch-Germanisches Museum, Köln)

\footnotetext{
${ }^{13}$ Die Abbildung bei ScHOPPA 1959, 64. Nr.80. Taf.72 zeigt bereits eine ausgemalte Inschrift.

${ }^{14}$ CIL XIII 4714.

${ }^{15}$ ILTG $533=$ AE 1927, 155.

${ }^{16}$ CIL XIII 1397.

${ }^{17}$ HARTLEY/DiCKINSON 2008a, 189-191.

18 2. N. 104-105. Nr.190. - Zu Anaillus s. KAKOSCHKe 2010, 208. CN 76. - Zum Pseudogentiliz Anaillius s. KAKOSCHKE 2006, 72-73. GN 76.
} 


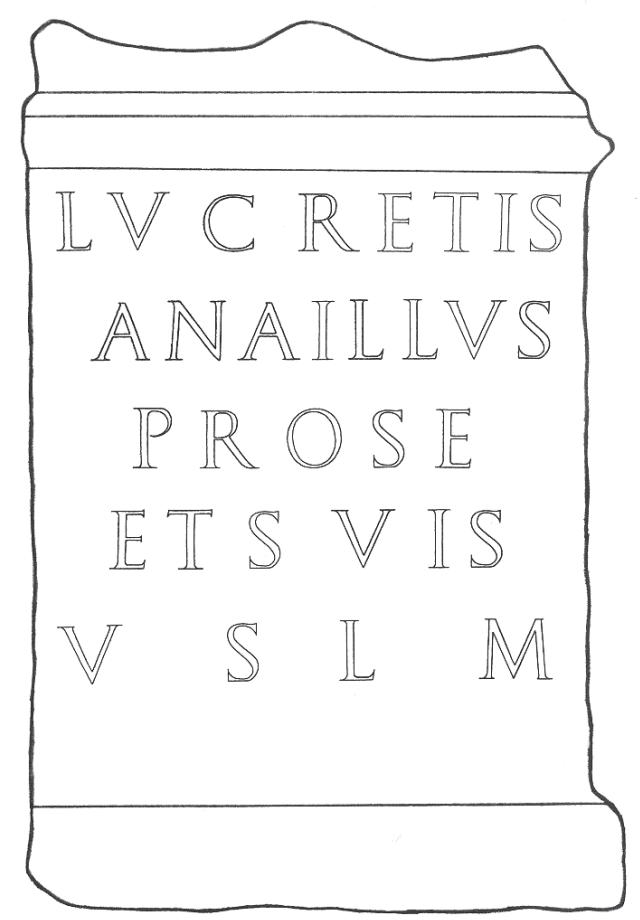

Abb.4: Leicht idealisierte Rekonstruktionszeichnung der Weihinschrift 1.N. 210-211. Nr.362 aus Köln/CCAA (Zeichnung A.K.)

Für die Kölner Inschrift wurde die Lesung des Namens ANAILLVS bereits von FREMERSDORF mit einem Fragezeichen vorgeschlagen. ${ }^{19}$ Allerdings geriet der Vorschlag offenbar in Vergessenheit. Entsprechend geben B. UND H. GALSTERER den Namen nur mit $A[---]$ wieder und bezweifeln zudem die korrekte Lesung der Hastenreste durch FREMERSDORF bzw. die korrekte Ausmalung der Hastenreste auf dem Objekt. ${ }^{20}$ Leider geben B. UND H. GALSTERER keine alternative Lesung des Namens in Zeile 2 an. Dies scheint angesichts des Zustandes der Inschrift auch kaum möglich. Sollte die Ausmalung der Hastenreste allein aufgrund der Lesung des Namens ANAILLVS durch FREMERSDORF erfolgt sein, wäre es auf jeden Fall nicht verwunderlich, dass die Hastenreste genau zu diesem Namen passen. Insofern bleibt die Lesung des Dedikatenamens selbstverständlich äußerst unsicher. Darüber hinaus ist auch der vorliegende Stein ohne Zweifel ein Argument gegen die nachträgliche moderne Ausmalung antiker Inschriften. ${ }^{21}$

III.

Im Jahre 1863 entdeckte man unweit des Herrenhauses Gripswald bei OssumBösinghoven (Stadt Meerbusch) zwölf teilweise stark fragmentarische Weihesteine. Die Steine, die den Matronae Octocannae bzw. in zwei Fällen dem Mercurius Arvernus geweiht waren, ${ }^{22}$ fanden sich alle innerhalb einer halbkreisförmigen Umfassungsmauer. Mit Sicherheit stammen die Tituli aus einem bei Ossum-Bösinghoven gelegenen Matronenheiligtum.

Zwei Jahre später kam bei Nachgrabungen am selben Ort der obere Teil eines weiteren Weihesteins zutage, der im Gegensatz zu den zuvor entdeckten Steinen heute

\footnotetext{
${ }^{19}$ F. FrEMERSDORF, Germania 13 (1929), 132. Nr.1.

${ }^{20}$ GALSTERER 1975, 27. Nr.77. Taf.17 (Photo), IKöln² 115-116. Nr.110.

${ }^{21}$ S. KAKoschKe 2014, 17-18 (mit Anm.21), 38. Anm.141, 41. Anm.168 (mit weiteren Hinweisen).

${ }^{22}$ CIL XIII 8570-8581.
} 
verloren ist. Die Inschrift ging in den Besitz von F. STOLLWERCK über. Diesem zufolge bestand der Stein aus „3 zusammengehörigen Bruchstücken“, die „über zwei Drittel des ganzen Steins" ausmachten (Abb.5). Die Maße des Steins gab STOLLWERCK mit $30 \mathrm{~cm}$ (Höhe), $24 \mathrm{~cm}$ (Breite) und $9 \mathrm{~cm}$ (Tiefe) an. Die Buchstaben hatten eine Größe von über $4 \mathrm{~cm}$. STOLLWERCK nahm an, dass der Altar ursprünglich etwa 40 bis $42 \mathrm{~cm}$ hoch war und im unteren Bereich noch eine oder zwei Zeilen gestanden hätten. Den Text des Weihesteins mit ,gut gemeißelten Buchstaben“ las STOLLWERCK unter Hinweis auf die Ligaturen in den Zeilen 2 (AN) und 4 (RI) wie folgt: ${ }^{23}$

Matronis / Octocan/abus Q. Va/rianus ...

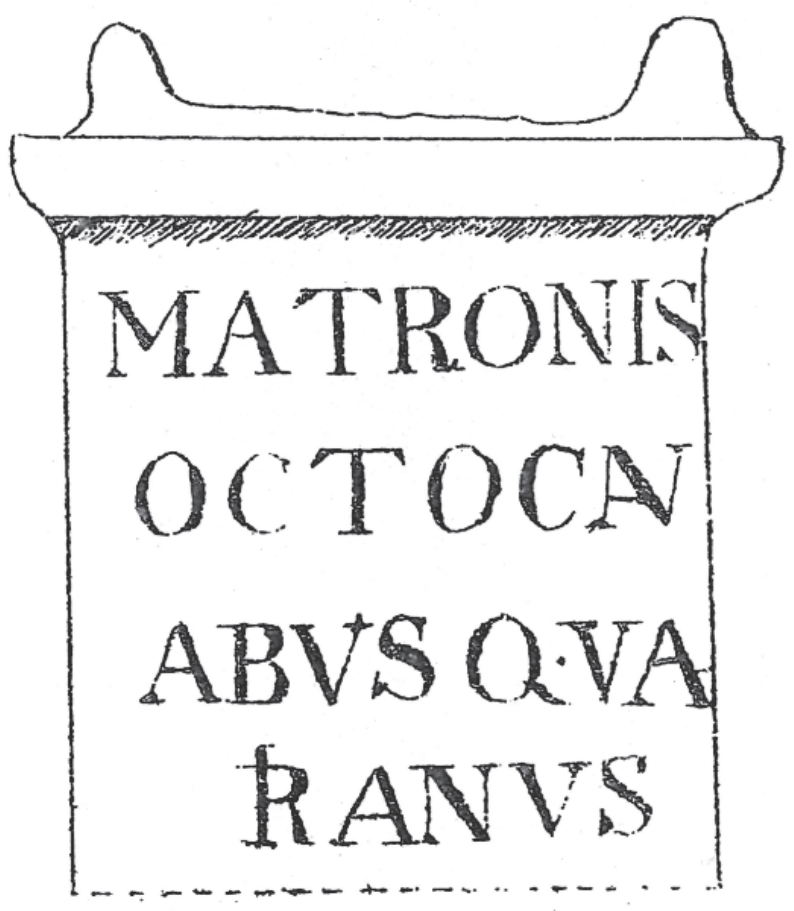

Abb.5: Fragmentarische Weihinschrift aus Ossum-Bösinghoven: CIL XIII 8574 (nach STOLLWERCK 1877, Taf.1)

Mit entsprechender Lesung nahm DOMASZEWSKI die Inschrift in den CIL-Band für die Germania inferior auf. ${ }^{24}$ Spätere Autoren und Datenbanken geben die Inschrift diesen Angaben folgend wieder, ${ }^{25}$ obwohl die korrekte Lesung der Inschrift bezweifelt werden darf.

STOLLWERCK erblickte in dem von ihm entzifferten Varianus ein Gentiliz. Für den Namen liegen jedoch nur zwei weitere Belege aus Italien vor. ${ }^{26}$ Dass der Dedikant aus Ossum-Bösinghoven, in dem man höchstwahrscheinlich einen Einheimischen erblicken kann, ein sehr seltenes italisches Gentiliz trug, ist zwar grundsätzlich nicht auszuschließen, jedoch eher unwahrscheinlich. Für die Lesung des Namens Q(uintinius) Varianus (oder ähnlich), könnte die Tatsache sprechen, dass das

\footnotetext{
${ }^{23}$ STOLLWERCK 1877, 173.

${ }^{24}$ CIL XIII 8574.

${ }^{25}$ RiESE 1914, 331. Nr.3164-4, GutenbrunNer 1936, 224. Nr.86-6, WeISGERBER 1968, 46. Nr.800, EDCS Nr.11100668.

${ }^{26}$ CIL VI 28332 (Rom), XI 5981 = I 2122 = VI 28389 (Cagli/Ad Calem). Zum Namen s. SchUlZE $1904,377$.
} 
vom Gentiliz Varius abgeleitete Varianus mehrmals als Cognomen bezeugt ist. ${ }^{27}$ Möglicherweise hat STOLLWERCK aber auch eine NI-Ligatur übersehen und auf dem Stein stand der Name Varianius, ein vom Cognomen Varianus abgeleitetes Pseudogentilitz, das grundsätzlich denkbar, bis heute jedoch nicht bezeugt ist.

Hier sei eine andere Lösung favorisiert. Schon IHM bezweifelte die Lesung STOLLWERCKS. „Nach der Zeichnung ... zu urtheilen“, stellte IHM fest, „könnte der Name des Dedikanten auch Va[le]rianus gelautet haben. " ${ }^{28}$ Sicherlich darf man mit IHM in RIANVS (wohl nicht IRANVS) mit einiger Berechtigung den Rest eines Cognomens erblicken. Da Q in Zeile 3 aber eher für das Pränomen Quintus steht als für ein abgekürztes Gentiliz, wird das folgende VA wahrscheinlich zu einem Gentiliz gehört haben. Es drängt sich daher die Vermutung auf, dass am Ende von Zeile 3 Val(erius) stand - mit einer vielleicht nur schlecht zu erkennenden AL-Ligatur. ${ }^{29} \mathrm{Am}$ Anfang von Zeile 4 fehlen, wie IHM aufgrund der Zeichnung STOLLWERCKs zu Recht vermutete, offenbar ein bis zwei Buchstaben. Entsprechend ließe sich der Name zu Aprianus, Arianus, Carianus, Syrianus, Verianus oder Varianus ergänzen. ${ }^{30}$ Denkbar ist auch Marianus mit einer MA-Ligatur. Wie die anderen aus Ossum-Bösinghoven bekannten Personen, in denen man romanisierte Einheimische vermuten darf, führte der Mann somit wohl die tria nomina mit einem gängigen Gentilnomen. ${ }^{31}$

Für die fragmentarische Weihinschrift aus Ossum-Bösinghoven sei somit zusammenfassend mit gebotener Vorsicht diese Lesung vorgeschlagen:

\author{
Matronis \\ Octocan- \\ abus $Q$ (uintus) $\bullet$ Val(erius) \\ [..] rianus \\ $5 \quad[v($ otum $) s$ (olvit) l(ibens) $m$ (erito)]
}

IV.

Zu den zahlreichen Weihesteinen, die in den Jahren 1928 und 1929 unter dem Bonner Münster entdeckt wurden, zählt ein oben leicht beschädigter Altar (Höhe $96 \mathrm{~cm}$ ) aus Sandstein für Mercurius Gebrinius (Abb.6). Die sauber eingeschlagene Inschrift des Steins aus der zweiten Hälfte des zweiten Jahrhunderts n. Chr. lautet unbestritten wie folgt: ${ }^{32}$

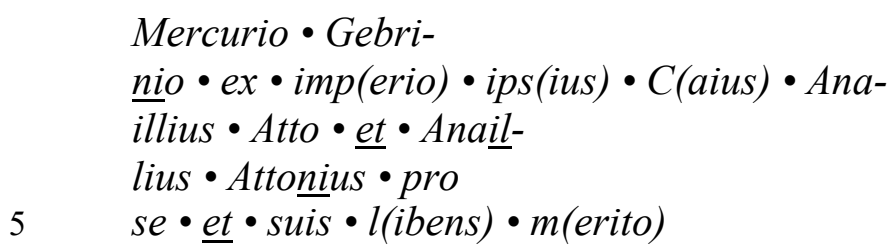

\footnotetext{
${ }^{27}$ S. auch KAKOSCHKE 2006, 416. GN 1352. - Zum Cognomen Varianus s. KAJANTO 1965, 158, OPEL IV 147 (mit dem Beleg aus Ossum-Bösinghoven).

${ }^{28}$ M. IHM, BJb 83 (1887), 154. Nr.327. Zweifel an der korrekten Lesung STOLLWERCKS hegte gleichzeitig auch M. SIEBOURG, WZ 6 (1887), 281, der jedoch keine alternative Lesung vorschlug.

${ }^{29}$ AL-Ligaturen können leicht übersehen werden, wie z.B. die Inschrift BRAMBACH 1867, 85. Nr.343 zeigt. Für den hier gelesenen Namen Cadinius bietet erstmals DüNTZER 1885, 41-42. Nr.41 die korrekte Lesung: Caldinius. - Mehr oder weniger deutliche AL-Ligaturen in Val(erius) bieten z.B. die Inschriften: CIL XIII 7987 (Wachtberg-Berkum), 3.NT 214. Nr.261 (Kapel Avezaath).

${ }^{30} \mathrm{Zu}$ den denkbaren Namen s. SOLIN/SALOMIES 1994², 461.

31 Die weiteren vollständigen Personennamen aus Ossum-Bösinghoven: CIL XIII 8571 (Q. Iulius Quietus, Q. Iulius Iucundus, Q. Iulius Ursulus), 8572 (C. Iulius Seranus, Vipsania Fahena), 8573 (C. Salvius Quetus), 8579 (M. Iulius Audax), 8580 (Sext. Sempronius Super).

32 2. N. 104-105. Nr.190 = H. LeHNER, BJb 135 (1930), 20. Nr.47. Taf.XXI (Photo). - Der Stein befindet sich heute im Rheinischen Landesmuseum in Bonn (Inv.-Nr.D276).
} 


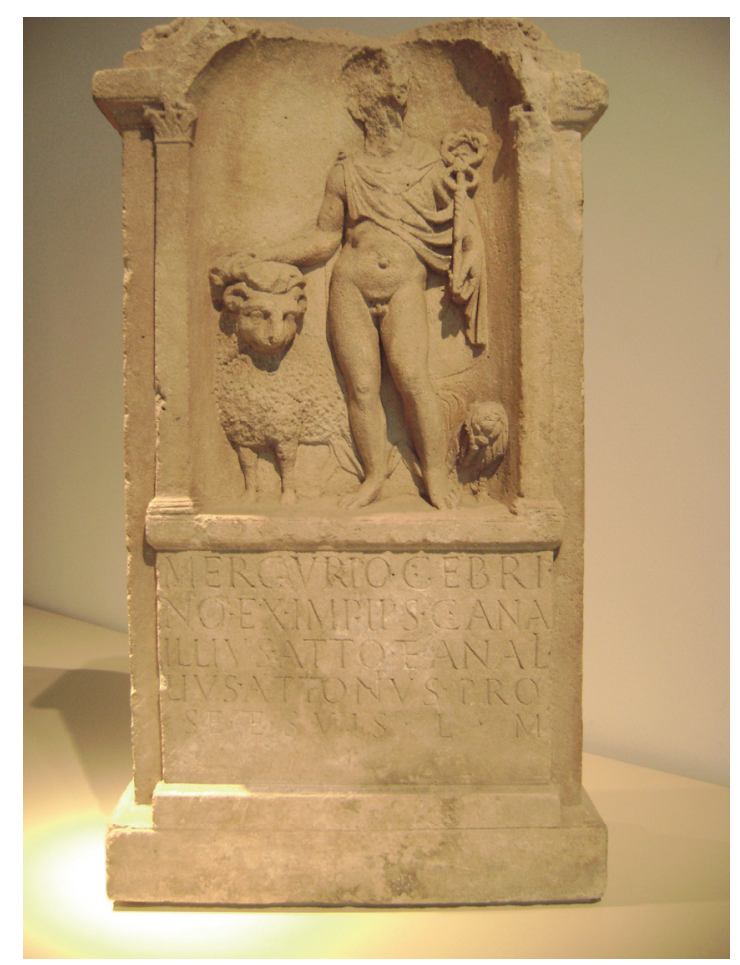

Abb.6: Weihinschrift aus Bonn/Bonna: 2. N. 104-105. Nr.190

(C) A.K.)

Leider geben die zwei Dedikanten, C. Anaillius Atto und Anaillius Attonius, ihr verwandtschaftliches Verhältnis nicht an. Höchstwahrscheinlich handelt es sich jedoch um Vater und Sohn. ${ }^{33}$ Offensichtlich hat der Sohn das aus dem keltischen Anaillus gebildete Pseudogentiliz des Vaters als Familiennamen übernommen. Zudem trägt er ein Cognomen, das vom Cognomen des Vaters, Atto, abgeleitet wurde. Attonius ist ansonsten allerdings nur als Pseudogentiliz bezeugt - und zwar vor allem in der Germania superior. ${ }^{34}$ Pseudogentilnomina wurden vom antiken Leser wohl stets als Patronymika empfunden, selbst wenn sie sich - wie im vorliegenden Fall der Name Anaillus - zu festen Familiennamen entwickelten konnten. ${ }^{35}$ In der Bonner Inschrift fungiert der wie ein Pseudogentiliz anmutende Name Attonius seiner Stellung nach eindeutig als Cognomen. Man darf nun sicherlich vermuten, dass in einer Gesellschaft, die mit Pseudogentilnomina vertraut war, Attonius als Pseudogentiliz bzw. als Patronymikon interpretiert wurde, selbst wenn es formal gesehen als Cognomen in Erscheinung trat. Dies gilt wohl um sehr mehr, wenn in einer Inschrift zwei Personen namens Atto und Attonius genannt wurden. Insofern darf man annehmen, dass der Name Anaillius Attonius vom einheimischen Leser (nicht nur, aber vor allem im entsprechenden Kontext) im Sinne von Anaillius, Sohn des Atto aufgefasst wurde. Diese patronymische Bildungsweise und Funktion von Cognomina ist bisher offenbar nicht näher thematisiert worden.

\footnotetext{
${ }^{33}$ In diesem Sinne äußern sich auch WeISGERBER 1968, 138, H. G. HorN, Das Leben im römischen Rheinland, in: RiNRW, 273. Abb.227 (Photo) und BAUCHHENß 2001, 331, 338. Nr.6-7. Vgl. dagegen J. HuPE, TZ 60 (1997), 147-148. Nr.19. Abb.11/2 (Photo), der in den Dedikanten Brüder vermutet.

${ }^{34}$ Zum Namen s. KAKOSCHKE 2006, 93. GN 159 (mit den Belegen).

35 Schulze 1904, 20, 23，53, Weisgerber 1968, 136, RAEPSAET-CHARLIER 2001, 410-413, A. KAKOSCHKE, FeRA 26 (2015), 188.
} 


\section{V.}

In Hürth-Gleuel (Rhein-Erft-Kreis), genauer in den Fundamenten der romanischen Pfarrkirche, fand sich im Jahre 1893 der Sockel (Höhe $89 \mathrm{~cm}$ - Breite $67 \mathrm{~cm}$ - Tiefe $65 \mathrm{~cm}$ ) einer Jupitersäule aus Kalkstein, der aus der Zeit zwischen 150 und 250 n. Chr. stammt. Der Stein (Abb.7) trägt folgende gut lesbare Inschrift: ${ }^{36}$
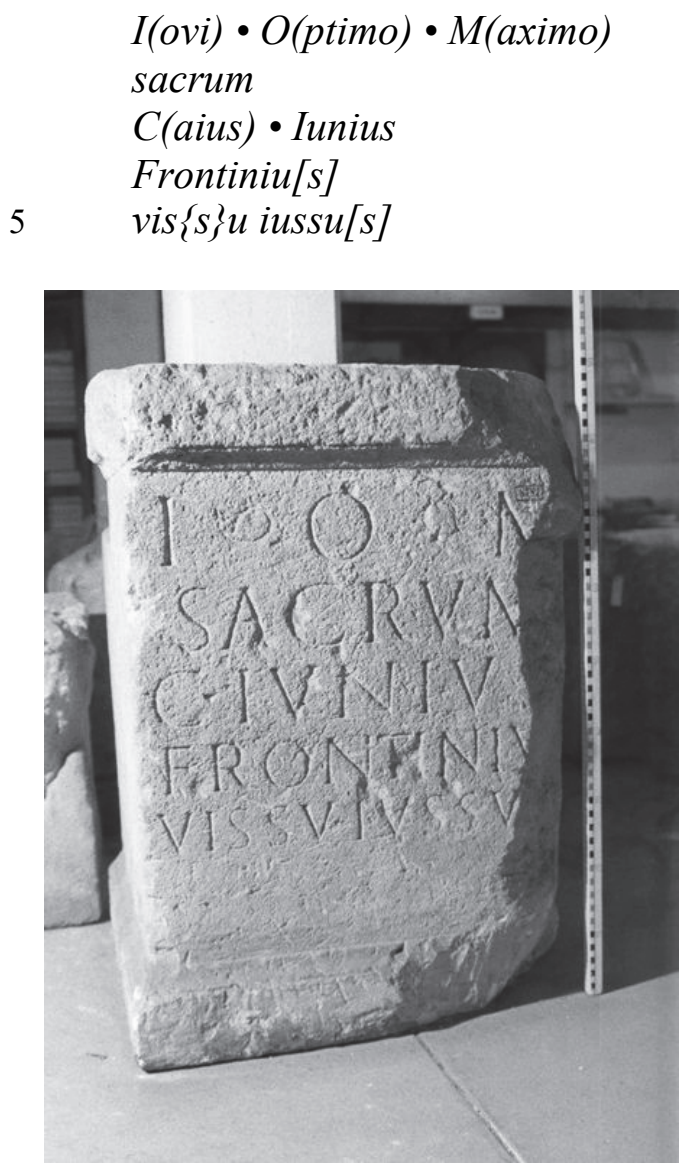

Abb.7: Weihinschrift aus Hürth-Gleuel: CIL XIII 8162

(C) CIL XIII/2-Projekt, Universität Flensburg/Trier)

Laut KLINKENBERG, dem Ersteditor der Inschrift, trug der Dedikant bemerkenswerterweise zwei Gentilnomina. ${ }^{37}$ Entsprechend führt LEHNER aus: „Der Stifter führt zwei Familiennamen, was auf Adoption oder dgl. beruhen wird. “38 Mit Sicherheit trug der Dedikant der Inschrift jedoch die tria nomina und Frontinius steht ohne Zweifel auf dem Platz des Cognomens. Insofern wird der Name in der neueren Forschung korrekterweise als Cognomen geführt. ${ }^{39}$ Das vom lateinischen Cognomen Frontinus oder Fronto abgeleitete Frontinius ist als Cognomen jedoch nicht weiter bezeugt, wohl aber als Pseudogentiliz. Als solches tritt der Name verstärkt in der Germania inferior, im näheren Umfeld von Hürth-Gleuel, auf. ${ }^{40}$ Insofern sind die Bemerkungen von KLINKENBERG und LEHNER verständlich. Und auch der antike Leser wird, ebenso

\footnotetext{
${ }^{36}$ CIL XIII 8162 = NOELKE 1981, 478. Nr.180. - Der Stein befindet sich heute im Rheinischen Landesmuseum in Bonn (Inv.-Nr.8787).

${ }^{37}$ J. KLINKENBERG, BJb 94 (1893), 154.

${ }^{38}$ LEHNER 1918, 26. Nr.49.

${ }^{39}$ KAJANTO 1965, 236, OPEL II 152, KAKOSCHKE 2007, 366. CN 1350.

${ }^{40}$ Zum Namen s. KAKOSCHKE 2006, 188. GN 509 (mit allen Belegen).
} 
wie im Fall des Anaillius Attonius (s.o.), das Cognomen als (bekanntes) Patronymikon interpretiert und den vollständigen Namen des Dedikanten als Gaius Iunius, Sohn des Frontinus oder des Fronto gedeutet haben. Man kann daher mit einiger Berechtigung davon ausgehen, dass der Vater des Mannes wahrscheinlich den Namen C. Iunius Frontinus oder Fronto getragen hat. Laut WEISGERBER entwickelten sich die aus der Germania inferior bekannten -inius-Namen verstärkt zu echten Sippennamen. ${ }^{41}$ Daher ist im vorliegenden Fall eine Auslegung des Namens im Sinne von Gaius Iunius aus der Familie der Frontinii nicht auszuschließen, wenn nicht gar wahrscheinlicher.

Wie die folgende (sicher unvollständige) Tabelle zeigt, finden sich in den zwei germanischen Provinzen weitere einheimische Personen mit duo oder tria nomina, die ein Cognomen führen, das ansonsten nicht weiter oder nur selten bezeugt ist. Die Cognomina erwecken den Eindruck eines Pseudogentilnomens und sind teilweise auch als Pseudogentilz anderweitig bezeugt. Da die Namen der Väter nicht bekannt sind, kann nur vermutet werden, dass die Cognomina der aufgeführten Personen vom Cognomen des Vaters abgeleitet wurden und ebenso wie ein Pseudogentiliz als Patronymikon fungierten oder im Fall eines -inius-Namens einen jungen Sippennamen anzeigen. Gewissheit herrscht nur im Fall der aus Metz/Divodurum bekannten Domit(ia) Sextia (s.u.), deren Vater, ein Veteran (aus dem Rheinland?), Q. Domitius Sextus hieß.

\begin{tabular}{|c|c|c|c|}
\hline Nr. & Nachweis & Fundort & Name \\
\hline 1 & CIL XIII 5929c & Bourbonne-les-Bains & Nivalius Agedinius \\
\hline 2 & CIL XIII 8788 & Domburg & Ianuarinius Ambacthius \\
\hline 3 & CIL XIII 8639 & Xanten-Birten/Vetera & Cessorinius Ammausius \\
\hline 4 & 1.N. 224 & Ober-Ingelheim & Miccionia Ammisia \\
\hline 5 & CIL XIII 7577 & Wiesbaden/Aquae Mattiacorum & Ulpius Arvatius \\
\hline 6 & CIL XIII 8654 & Xanten-Birten/Vetera & M. Ulp(ius) Aspadius \\
\hline 7 & 2. N. Nr.190 & Bonn/Bonna & Anaillius Attonius (s.o.) \\
\hline 8 & CIL XIII 12026 & Pesch & Iulius Capi[ton]i[us](?) \\
\hline 9 & CIL XIII 12034a & Pesch & Pompi[a] Ce[leri]ni[a](?) \\
\hline 10 & 1. N. Nr.287 & Hürth-Hermühlheim & Q. Cottius [C]olonius \\
\hline 11 & CIL XIII 8521 & Dormagen-Gohr & Celsinius Cumius \\
\hline 12 & CIL XIII 11946 & Heddernheim/Nida & Sollius Decuminius \\
\hline 13 & CIL XIII 7932 & Zülpich-Geich/Tolbiacum & Octaviu[s E]xomnius \\
\hline 14 & CIL XIII 6318 & Rastatt-Wintersdorf & Iustin[i]a F[e]lic[i]a \\
\hline 15 & CIL XIII 2633 & Aubigny-la-Ronce & Tertinia Florentinia $^{42}$ \\
\hline 16 & CIL XIII 8525 & Dormagen/Durnomagus & Iulia Frapia \\
\hline 17 & CIL XIII 8162 & Hürth-Gleuel & C. Iunius Frontiniu[s] (s.o.) \\
\hline 18 & CIL XIII 7122 & Mainz/Mogontiacum & Vitalinius Gromatius \\
\hline 19 & AE 1977,570 & Xanten-Wardt/CUT & [Iu]n(ius) Hetinius \\
\hline 20 & CIL XIII 7858 & Eschweiler & Ulpius Hunicius \\
\hline 21 & CIL XIII 6776 & Mainz/Mogontiacum & M. Biracius Indutiu[s] \\
\hline 22 & IKöln² Nr.511 & $\mathrm{Köln} / C C A A$ & Sex. Lae[vi]nius Lautinius \\
\hline 23 & CIL XIII 7589 & Wiesbaden/Aquae Mattiacorum & [---]us Novellius \\
\hline 24 & CIL XIII 6636 & Stockstadt & G. Iul(ius) Petunnatius \\
\hline 25 & CIL VI 3335 & Rom & M. Callienius Placidinius ${ }^{43}$ \\
\hline 26 & CIL XIII 7520 & Bingen-Bingerbrück & Iulia Quintia \\
\hline 27 & AE $1969 / 70,442$ & $\mathrm{Köln} / C C A A$ & Tiberius Cl(audius) Romanius \\
\hline 28 & CIL XIII 8612 & Xanten/CUT & M. Hilarinius Romanius \\
\hline 29 & CIL XIII 7555a & Hinzerath/Belginum & C. Vellorius Sacrillius \\
\hline 30 & $\mathrm{AE} 1978,566$ & Bern & [O]tacil(ius) Seccius \\
\hline 31 & CIL XIII 6013 & Brumath/Brocomagus & Aelius Segileius \\
\hline
\end{tabular}

\footnotetext{
${ }^{41}$ WeISGERBER 1968, 137-138.

${ }^{42}$ Die junge Frau war eine Neckarsuebin.

${ }^{43}$ Der Mann war ein benef(iciarius) legat(i) leg(ionis) I Minervi(ae).
} 
Frankfurter elektronische Rundschau zur Altertumskunde 32 (2017)

\begin{tabular}{|l|l|l|l|}
\hline 32 & 1.NT 184 + 185 & Dieburg & Priscinius Sedulius \\
\hline 33 & Kolbe 1960, Nr.2 & Morken-Harff & M. Antonius Sentius \\
\hline 34 & CIL XIII 4330 & Metz/Divodurum & Domit(ia) Sextia (s.o.) \\
\hline 35 & CIL XIII 7678 & Maifeld-Polch & Iul(ia) Suausia \\
\hline 36 & CIL XIII 7911 & Nideggen-Embken & T. Iulius Suhetius \\
\hline 37 & CIL XIII 5048 & Valeyres & T. Capitonius Surdonedonius \\
\hline 38 & AE 1991, 1255 & Avenches/Aventicum & Sex(tius?) Tetricius \\
\hline 39 & CIL XIII 8601 & Geldern-Pont & [P]rimini[us] Tullius \\
\hline 40 & AE 1984, 668 & Bonn/Bonna & M. Aur(eliu)s Tutorius \\
\hline 41 & CIL XIII 3624 & Namur/Namurcum & Ulp(ia) Vanaenia \\
\hline 42 & CIL XIII 5311 & Munzach & C[1(audia)] Victorini[a] \\
\hline
\end{tabular}

Tabelle 1: Belege für die Namensform „(Pränomen +) (Pseudo-)Gentiliz + Cognomen auf -(in)ius/ -ia" für Personen aus den zwei germanischen Provinzen

Die genannten Namen sind wohl rein formal bzw. aus römischer Sichtweise als Cognomina zu bezeichen. Cognomina auf -ius sind auch nicht ungewöhnlich. Allerdings zeigen die hier aufgeführten Cognomina möglicherweise eine andere semantische Füllung als gängige Cognomina. Ihre denkbare patronymische Funktion rückt sie in die Nähe von Pseudogentilnomina. Insofern stellen die Namen eine Mischform zwischen Gentil- und Cognomina dar.

Das beschriebene Phänomen ist sicherlich der Ausdruck einer romanisierten einheimischen Gesellschaft, die zwar das römische Namenssystem weitgehend übernommen hat, aber andereseits an der keltisch-germanischen Sitte festhielt, den Individualnamen des Vaters oder den Namen der Sippe im eigenen Namen zu nennen. Die beschriebene Namensform zeigt einen flexiblen und innovativen Umgang mit dem römischen Namensystem, das den eigenen Bedürfnissen angepasst wurde.

Namen wie Anaillius Attonius oder C. Iunius Frontinius sind somit ebenso als Neuschöpfungen anzusehen wie die unrömischen Namen Cassius Verecundinius Firmus oder M. Iulius Vassileni f. Leubo, ${ }^{44}$ also Namen der Form „Gentiliz + Pseudogentiliz + Cognomen“ bzw. „Gentiliz + cognominale Filiation + Cognomen“. ${ }^{45}$ Wie die neu kreierten Pseudogentilnomina ${ }^{46}$ sind diese Namen, die fast ausnahmslos aus dem zweiten und dritten Jahrhundert n. Chr. stammen, als eine bemerkenswerte Erfindung der einheimischen romanisierten Gesellschaft der Rheinlande zu werten.

VI.

Bei St. Severin in Köln/CCAA befand sich eine römische Grabinschrift, deren Text nur handschriftlich durch Angaben aus dem 18. bzw. frühen 19. Jahrhundert überliefert ist. Im CIL wird die Inschrift aufgrund der Abschrift LAMEYs wie folgt wiedergegeben:

\footnotetext{
${ }^{44}$ CIL XIII 11982 (Remagen/Rigomagus), KolBE 1960, 57-58. Nr.5 (Morken-Harff).

${ }^{45} \mathrm{Zu}$ den genannten Namensformen s. KAKOSCHKE 2002, 360, A. KAKOSCHKE, GFA 18 (2015), 182183, ders., FeRA 30 (2016), 8-13 (jeweils mit weiteren Hinweisen und Literaturangaben).

${ }^{46}$ Zur Beurteilung der Pseudogentilnomina s. A. KAKOSCHKE, FeRA 26 (2015), 14-17, ders., FeRA 30

(2016), 9-10 (jeweils mit weiteren Hinweisen und Literaturangaben).
} 


\title{
DIG N IO
}

\section{QVARTO \\ APRONO}

BVB A LVS

\section{FRATRI $\cdot$ F}

\author{
Abb.8: Grabinschrift aus Köln/CCAA: CIL XIII 8378 \\ (nach CIL XIII/2.2. 578. Nr.8378)
}

Dagegen las HüPSCH in Zeile 3 Apronius und am Ende von Zeile 5 statt $\mathrm{F}$ für fecit $\mathrm{P}$ für posuit. ${ }^{47}$ Entsprechend gibt das CIL im Kommentar zur Inschrift für Zeile 3 APRONIVS an und im Index des CIL findet man den Namen Apronius Bubalus. ${ }^{48}$ Das OPEL dagegen führt Apronio(!) als Cognomen auf. ${ }^{49}$ B. und H. GALSTERER übersetzten die Inschrift mit den Worten „Für Dignius, Quartus und Apronius. Bubalus hat (die Grabinschrift) für seinen Bruder (sic) aufgestellt", wobei sie davon ausgehen, „daß in der letzten Zeile ,fratrib(us)“ stand.“" ${ }^{60}$ Sinnvoller ist dagegen die Annahme Solins, dass LAMEY den Namen in Zeile 3 falsch überliefert hat. Solin folgt $\mathrm{HÜPSCH}$, demzufolge die Inschrift von Apronius Bubalus für seinen Bruder Dignius Quartus gesetzt wurde. Gleichzeitig muss er jedoch eingestehen, dass Apronius zumindest theoretisch auch ein zweites Cognomen des Dignius Quartus darstellen könnte. ${ }^{51}$

Zur Vorsicht mahnt die Tatsache, dass, wie auch SOLIN einräumt, LAMEY in der Forschung als der zuverlässigere Autor gilt. ${ }^{52}$ So gibt LAMEY zur Inschrift mehr Informationen als HÜPSCH. Er übermittelt den Aufbewahrungsort der Inschrift, bei St. Severin, und verzeichnet eine NI-Ligatur in Apronio. Aus diesem Grund gab wohl DOMASZEWSKI im CIL die Lesung LAMEYs wieder. Weniger Bedeutung hat der Umstand, dass nach der favorisierten Lesung Solins die Brüder unterschiedliche Gentilnomina tragen. Schließlich wurde der Begriff frater, wie B. und H. GALSTERER zutreffend feststellen, nicht immer im wörtlichen Sinne verwendet.

Geht man davon aus, dass die ältere Lesung LAMEYs korrekt ist, hieß der Verstorbene Dignius Quartus Apronius. SolIN zufolge ist ein solcher Name kaum vorstellbar. Allerdings finden sich in den zwei germanischen Provinzen im zweiten und dritten Jahrhundert n. Chr. vergleichbare dreigliedrige Namen, wie Cassius

\footnotetext{
${ }^{47}$ HÜPSCH 1801, 20. Nr.71.

${ }^{48}$ CIL XIII/5 3 (Index).

49 OPEL I ${ }^{2} 69$.

${ }^{50} \mathrm{IKöln}^{2} 379$. Nr.458.

${ }^{51}$ H. Solin, Arctos 32 (2003), $190=$ AE 2003, 1217.

${ }^{52}$ H. Solin, Arctos 32 (2003), 190 unter Verweis auf CIL XIII/2,2 p. 508-509.
} 
Verecundinius Firmus oder Martius Senopatius Novellus ${ }^{53}$ Die weiter oben bereits erwähnte Namensform läßt sich durch die Formel „Gentiliz + Pseudogentiliz + Cognomen" beschreiben und entspricht der ebenfalls oftmals bezeugten Namensvariante „Gentiliz + cognominale Filiation + Cognomen“. Dazu treten Namen mit einer nachgestellten cognominalen Filiation, also der Form „Gentiliz + Cognomen + cognominale Filiation“.${ }^{54}$ Eine hierzu passende Variante der Form „Gentiliz + Cognomen + Pseudogentiliz" scheint bisher nicht bekannt zu sein. Immerhin trifft man in Budapest/Aquincum eine Person namens Sep(timius) Colonus Attusonius. ${ }^{55}$ Der Name könnte die Leerstelle füllen, da es sich bei Attusonius offensichtlich um ein Pseudogentiliz mit keltischer Basis handelt. In der Form Atusonius ist der Name als Pseudogentiliz auch in Jagsthausen bezeugt. ${ }^{56}$ Ferner lässt z.B. auf die Mithras-Weihung eines Senilius Carantinus sive Cracissius aus Heddernheim/Nida verweisen. ${ }^{57}$ Bisher sah man in Cracissius stets einen religiösen Beinamen. ${ }^{58}$ Cracissius kann man aber ebenso als patronymische Bildung zum keltischen Namen Cracissa auffassen. ${ }^{59}$ Der Mann trug also möglicherweise einen Namen der Form „(Pseudo-)Gentiliz + Cognomen + Pseudogentiliz“.

Schließlich lässt sich der Name in der Kölner Inschrift entsprechend einstufen. Bei Apronius kann es sich durchaus um ein vom lateinischen Cognomen Apro oder Aper abgeleitetes Pseudogentiliz handeln. ${ }^{60}$ Somit wäre der Name gleichbedeutend mit Dignius Quartus Apronis/Apris filius.

\section{VII.}

Aus Köln/CCAA, der genaue Fundort und das Fundjahr sind unbekannt, stammt ein links und unten abgebrochener Block (Höhe $37 \mathrm{~cm}$ - Breite $50 \mathrm{~cm}$ - Tiefe $46 \mathrm{~cm}$ ) aus Kalkstein, der offenbar zu einer Grabinschrift gehört (Abb.9). Das Inschriftfeld wird von einem Blattrahmen eingefasst. B. und H. GALSTERER, die das Fragment in das erste bis zweite Jahrhundert n. Chr. datieren, geben die Inschrift des Steins in Umschrift wie folgt wieder: ${ }^{61}$

\section{C(aius) $\bullet$ Paternius \\ Cantabr(us) \\ Caiae •}

Gemäß dieser Lesung nennt die Inschrift in den ersten beiden Zeilen einen C. Paternius Cantabrus. B. und H. GALSTERER fügen erläuternd hinzu: „Wahrscheinlich hieß der Verstorbene ,Paternius', zumindest ist eine NI-Ligatur in Z. 1 aufgrund des

\footnotetext{
${ }^{53}$ CIL XIII 11982 (Remagen/Rigomagus), 2.N. 78. Nr.88 (Alzey/Altiaia).

${ }^{54}$ Ausführlicher hierzu A. KAKOSCHKE, FeRA 30 (2016), 8-13.

${ }^{55}$ CIL III $10557=$ LUPA Nr.2971.

${ }^{56}$ CIL XIII 6554.

${ }^{57}$ CIL XIII $7369=$ KAKOSCHKE 2002, 99-100. Nr.1.71.

${ }^{58}$ Zum Namen s. KAKOSCHKE 2007, 270. CN 946 (mit weiteren Hinweisen).

${ }^{59} \mathrm{Zu}$ Cracissa s. HARTLEY/Dickinson 2008b, 166-167 (mit einem Töpfer dieses Namens aus Lezoux in Aquitanien).

$60 \mathrm{~S}$. in diesem Zusammenhang den bemerkenswerten Namen C. Apronius Aper aus BornheimSechtem (CIL XIII 8153).

${ }^{61}$ IKöln $^{2}$ 429-430. Nr.547 (mit Photo). Worttrenner und Ligaturen wurden hier gemäß den kommentierenden Informationen zur Inschrift bzw. gemäß einer älteren Publikation [GALSTERER 1972/73, 99. Nr.18. Taf.36/1 (Photo)] ergänzend angegeben. Der Lesung folgt die EDCS Nr.00500109. - Das Objekt befindet sich heute im Römisch-Germanischen Museum in Köln (Inv.-Nr.74, 462).
} 
tiefer ansetzenden V nicht auszuschließen.“ In Zeile 3 steht dann offenbar der Name einer Frau im Dativ. Die Autoren vermerken zu ihrer Lesung jedoch einschränkend: „,Caia' als Praenomen einer Frau zu interpretieren, wäre höchst spekulativ.“

Diese Lesung bzw. Interpretation der fragmentarischen Inschrift kann m.E. so nicht stimmen. Zunächst ist festzustellen, dass in der ersten Zeile eindeutig PATERNVS steht, nicht PATERNIVS. ${ }^{62}$ Das V wurde vermutlich nur tiefer angesetzt, um Platz zu sparen. An der rechten Vertikalhaste des N sind keine weiteren tiefer sitzenden Serifen zu erkennen, die für eine NI-Ligatur sprechen könnten. Am Anfang der letzten Zeile ist noch der obere Teil eines $\mathrm{C}$ oder eines $\mathrm{G}$ zu erkennen. B. und H. GALSTERER haben diesen Buchstaben in zwei älteren Publikationen auch noch angegeben. ${ }^{63}$ Im Folgenden sollte nicht CAIAE sondern CALAE bzw. GALAE gelesen werden, da die Laufweiten der Buchstaben eher für L als für ein I sprechen. Der Platz für die Waagerechte des L ist jedoch knapp bemessen. Entsprechend halten auch B. und H. GALSTERER hier ein ,etwas weiter heruntergezogenes ,L“" für möglich. ${ }^{64}$ Ein Wortrenner hinter CALAE ist nicht zu erkennen. Schließlich darf man entgegen der Auffassung von B. und H. GALSTERER bezweifeln, dass die erhaltenen Zeilen komplett vorliegen. Sicher fehlen auf der linken Seite noch weitere Buchstaben. Hierfür spricht vor allem die letzte Zeile, in der das C vor CALAE als erster Buchstabe in der Zeile nicht plausibel zu deuten ist. ${ }^{65} \mathrm{Zudem}$ liegen die Anfangsbuchstaben am vermeintlichen Beginn der Zeilen nicht auf einer Höhe. Es ist daher zu bezweifeln, dass das $\mathrm{C}$ der ersten drei Zeilen jeweils am Zeilenbeginn stand. ${ }^{66}$

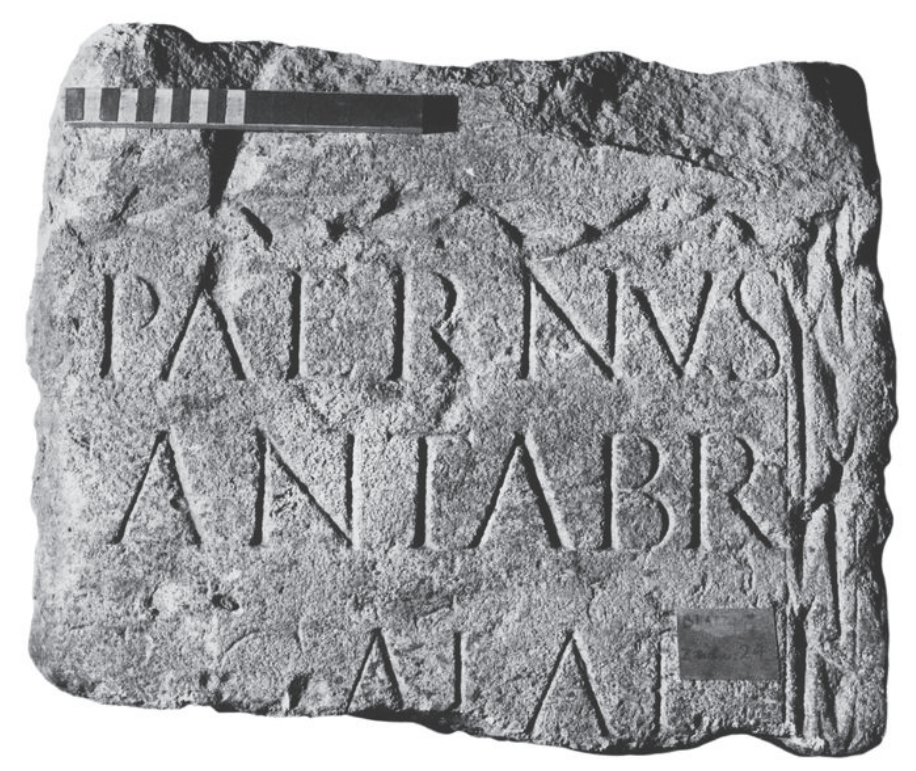

Abb.9: Fragmentarische Grabinschrift aus Köln/CCAA: IKöln² 429-430. Nr.547

(C) Römisch-Germanisches Museum, Köln)

\footnotetext{
${ }^{62}$ S. auch noch Galsterer 1972/73, 99. Nr.18. Abb.36/1 (Photo) und GAlsterer 1975, 86. Nr.372. Taf.83 (Photo). Vgl. ferner KAKOSCHKE 2002, 337-338. Nr.3.6.

${ }^{63}$ Galsterer 1972/73, 99. Nr.18. Abb.36/1 (Photo) und Galsterer 1975, 86. Nr.372. Taf.83 (Photo).

${ }^{64} \mathrm{IKöln}^{2}$ 429-430. Nr.547 (mit Photo).

${ }^{65}$ In diesem Sinne auch GALSTERER 1972/73, 99. Nr.18. Abb.36/1 (Photo) und GALSTERER 1975, 86. Nr.372. Taf.83 (Photo).

${ }^{66}$ Eine entsprechende Auffassung spiegelt auch noch die Lesung GALSTERER 1972/73, 99. Nr.18. Abb.36/1 (Photo) wieder: --- C • PATERNVS / ----- CANTABR / ---- C CAIAE • / ------ (= AE 1974, 460).
} 
Über diese Kritik hinaus lässt sich zu der fragmentarischen Inschrift allerdings kaum etwas Sicheres sagen. Möglicherweise kann in der ersten Zeile C(ai filius) Paternus gelesen werden. CANTABR gehört vielleicht zu einer Herkunftsangabe, etwa [ex gente] Cantabr/[orum] oder Cantabr(o) / Iuliobrigensi. Denkbar ist aber ferner die Ergänzung $\mathrm{zu}$ einem Cognomen, also vielleicht $\mathrm{zu}$ Cantabr/[inus] oder Cantabr/[iacus], sofern nicht Cantab(e)r (ethnische Herkunftsangabe oder Cognomen) zu lesen ist. ${ }^{67}$ Die von B. und H. GALSTERER gelesene maskuline Form Cantabr(us) ist auf jeden Fall nicht weiter bezeugt. ${ }^{68}$ Aufgrund der Stationierungsgeschichte lässt sich eine noch mögliche Lesung wie [coh(ortis) II] Cantabr/[orum] oder ähnlich eher ausschließen. ${ }^{69}$

Stimmt die vorgeschlagene Lesung der letzten Zeile, kann hier fast nur zu C(ai filius) Calae/[tus] oder C(ai filio) Calae/[to] ergänzt werden. Dies ist insofern bemerkenswert, als der Name Calaetus fast nur in Hispanien bezeugt ist. ${ }^{70}$ Sowohl das offenbar unvollständige CANTABR als auch Calaetus deuten somit Richtung Hispanien.

\section{VIII.}

Im Jahre 1845 entdeckte man im Cäcilienhospital (heute Josef-Haubrich-Hof) in Köln/CCAA eine schlecht erhaltene Weihinschrift für Mercurius Arvernus. Der Altar aus Kalkstein (Höhe 31,5 cm - Breite $22 \mathrm{~cm}$ - Tiefe $13 \mathrm{~cm}$ ) ist an der rechten Seite und unten nicht mehr vollständig erhalten (Abb.10). B. und H. GALSTERER, die den Stein in das zweite bis dritte Jahrhundert $\mathrm{n}$. Chr. datieren, geben die teilweise stark abgeriebene Inschrift des Steins älteren Lesungen entsprechend wie folgt wieder: ${ }^{71}$

$$
\begin{array}{ll} 
& \text { Mercur }[\text { io }] \\
& {[\text { A]rvern }[o]} \\
& {[\text { s]acru }[\mathrm{m}]} \\
& [\text { I] }] \text { ulius Iu[...] }] \\
5 \quad & {[\text { e }] \text { ximp }(\text { erio }) \text { i }[\text { ps }(\text { ius })]}
\end{array}
$$

Zum Namen des Dedikanten heißt es im Kommentar: „,..., so daß vom Namen des Dedikanten nur Iulius und die ersten beiden Buchstaben des Cognomens zweifelsfrei lesbar sind. Das Cognomen war wohl sicher abgekürzt." In diesem Sinne hatte bereits DÜNTZER vorgeschlagen, den Namen als Iulius Iu(sa) oder Iulius IV (= Quartus) zu lesen. ${ }^{72}$ Dementsprechend gab DoMASZEWSKI im CIL, im Kommentar zur Inschrift, die Lesung [I]ulius Iu[..] an. ${ }^{73}$ Ein abgekürztes Cognomen liegt auf jeden Fall insofern nahe, als ein mit IV beginnendes Cognomen, das insgesamt nur aus wenigen Buchstaben besteht, schwer zu finden ist. Sieht man von sehr seltenen Namen ab, bietet sich aus dem Material der in den germanischen Provinzen bezeugten Namen

\footnotetext{
${ }^{67} \mathrm{Zu}$ Cantabrinus [CIL II 5495 (Alomartes)] bzw. Cantabriacus [CIL III 4471 (Petronell/Carnuntum)] s. OPEL II 32. Zu Cantaber s. OPEL II 32, KAKOSCHKE 2007, 201-202. CN 647. - KAJANTO 1965, 198 gibt noch einen Beleg für das Cognomen Cantabrius [CIL VI 27441 (Rom)] an. Solin/SALOMIES $1994^{2}$, 45 führen den Beleg Cantabriacus (s.o.) als Gentiliz an sowie einen weiteren Nachweis für das Gentiliz Cantabrius aus Rom (EDCS Nr.39900248).

${ }^{68}$ S. auch OPEL II 32, das den Beleg aus Köln unter dem Lemma CANTABR* als Cantabr() führt.

${ }^{69}$ SPAUL 2000, 99-100.

${ }^{70} \mathrm{Zu}$ Calaetus s. OPEL II 22.

${ }^{71}$ IKöln$^{2}$ 160-161. Nr.173. - Der Weihestein befindet sich heute im Römisch-Germanischen Museum in Köln (Inv.-Nr.371).

72 DÜNTZER 1885, 25-26. Nr.9.

${ }^{73}$ CIL XIII 8235. S. auch CIL XIII/5 10 (Index).
} 
nur Iullus an, das manchmal mit nur einem L eingeschlagen wurde. ${ }^{74}$ Allerdings scheint der Platz für IVLVS kaum auszureichen, sofern der Steinmetz nicht am Ende der Zeile mit Ligaturen gearbeitet hat. Fehlende Ligaturen im erhaltenen Schriftbild sprechen jedoch eher gegen diese Möglichkeit.

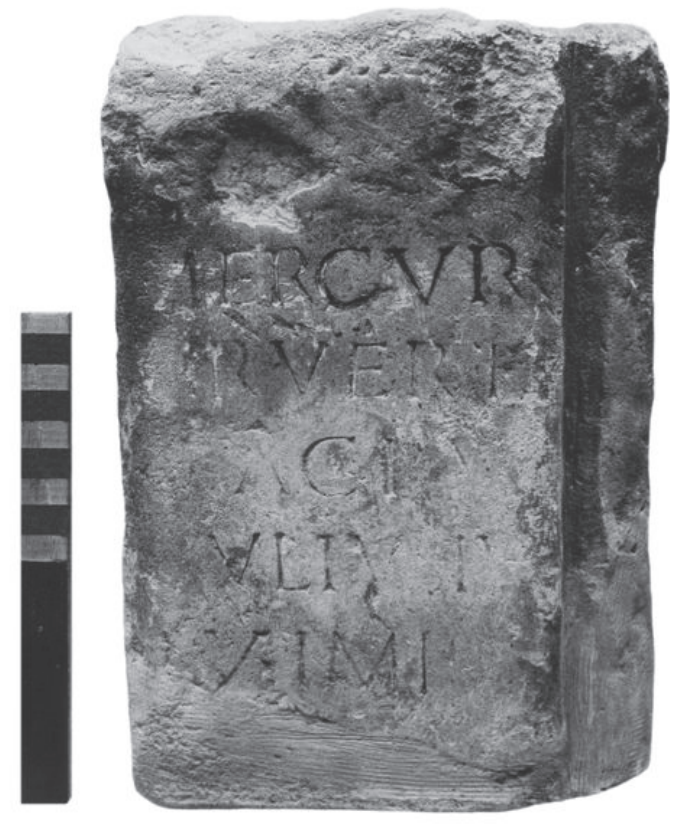

Abb.10: Weihinschrift aus Köln/CCAA: CIL XIII 8235

(C) Römisch-Germanisches Museum, Köln)

Da abgekürzte Cognomina im Vergleich zu abgekürzten Gentilnomina in Steininschriften viel seltener auftreten und die Datierung der Inschrift ohnehin für eine abgekürzte Form des gängigen Gentilnomens Iulius spricht, liegt m.E. in der vierten Zeile die Lesung IVL IVSIV nahe. Dabei können die noch zu lesenden Hasten des Cognomens offenbar nur zu dem ebenfalls äußerst gängigen Iustus gehören. ${ }^{75}$ Bei dem vermeintlichen I wird es sich wohl um ein abgeriebenes T handeln, das oftmals etwas größer eingeschlagen wurde, so dass die waagerechte Haste platzsparend über den angrenzenden Buchstaben stand.

Abschließend sei noch darauf verwiesen, dass sich im epigraphischen Material auch Belege für ein fehlerhaft eingeschlagenes IVSIVS (statt IVSTVS) oder IVSIINVS (statt IVSTINVS) finden. ${ }^{76}$

\section{IX.}

In der Kasinostraße (südliche Ecke Pipinstraße) in Köln/CCAA entdeckte man im Jahre 2003 die linke obere Ecke eines Grabsteins (Höhe $30 \mathrm{~cm}$ - Breite 22,5 cm Tiefe 8,5 cm) aus Kalkstein (Abb.11). Die erhaltenen Buchstaben weisen eine teilweise noch gut erkennbare rote Ausmalung auf. Die Ersteditoren B. und

\footnotetext{
${ }^{74} \mathrm{Zu}$ Iullus s. OPEL II 207, KAKOSCHKE 2007, 436-437. CN 1638.

${ }^{75} \mathrm{Zu}$ Iustus s. OPEL II 210, KAKOSCHKE 2007, 441-443. CN 1651.

${ }^{76}$ Für die zwei germanischen Provinzen s. CIL XIII 7399 (Friedberg) und 8772 (Ruimel) - vielleicht ein Lese- bzw. Überlieferungsfehler. - Grundsätzlich zu entsprechenden Fehlern in Inschriften aus den zwei germanischen Provinzen s. KAKOSCHKE 2014, 42-43.
} 
H. GALSTERER, die das Fragment in das zweite Jahrhundert $n$. Chr. datieren, geben die Inschrift des Steins in Majuskelschrift wie folgt wieder: ${ }^{77}$

$$
\begin{aligned}
& D \text { [M] } \\
& \text { FUSC[---] } \\
& \text { GRI[---] } \\
& \text { OSU[---] }
\end{aligned}
$$

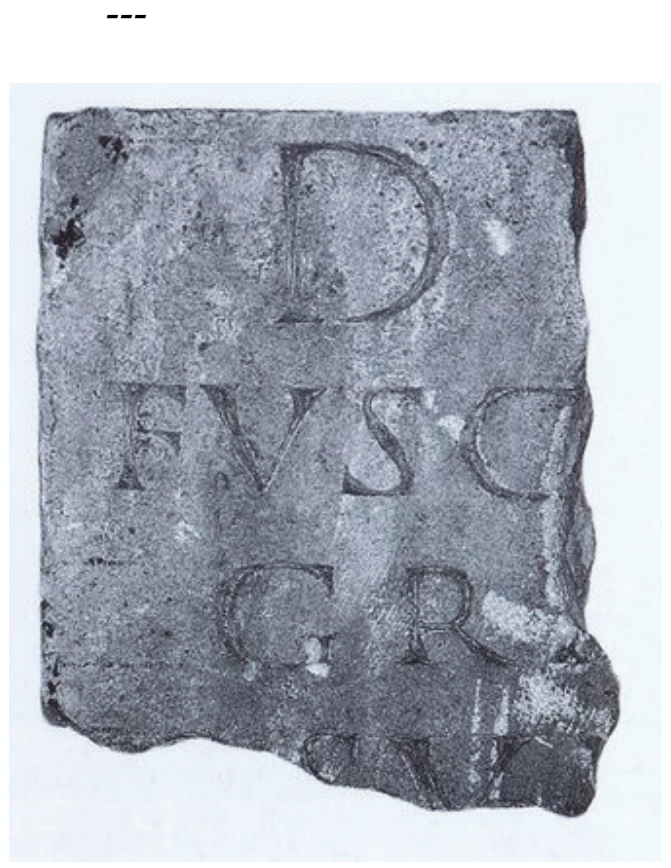

Abb.11: Fragmentarische Grabinschrift aus Köln/CCAA: AE 2010, 1014

(C) Römisch-Germanisches Museum, Köln)

In Zeile 2 schlagen B. und H. GALSTERER zu Recht eine Ergänzung zum Gentiliz Fuscinius vor, erwägen aber ebenso die Lesung Fusc[ianius]. Für das in Zeile 3 entzifferte GRI schwanken die Autoren zwischen einer Ergänzung zu Grittius oder zu dem als Namen nicht bezeugten Grillus. Schließlich lässt sich OSU, B. und H. GALSTERER zufolge, am einfachsten zu posuit vervollständigen.

Eine sichere Ergänzung des Textes ist bestimmt nicht möglich, dennoch soll hier eine alternative Lesung vorgeschlagen werden: Bei dem noch im Ansatz zu erkennenden Buchstaben in Zeile 3 handelt es sich m.E. nicht um ein I, sondern um ein A. Es besteht also kein Anlass, nach einem sehr seltenen mit Gri- beginnenden Cognomen zu suchen. ${ }^{78}$ Da in Zeile 2 höchstwahrscheinlich das Pseudogentiliz Fuscinius stand, für das zwei weitere Belege aus Niedergermanien vorliegen, ${ }^{79}$ sprechen die Platzverhältnisse in Zeile 3 (Abb.12) für die Lesung des nicht nur in der Germania inferior äußerst gängigen Cognomens Gratus. ${ }^{80}$ Bei der genannten Person wird es sich um den Verstorbenen gehandelt haben, dessen Name, wie in Köln/CCAA und Nieder-

\footnotetext{
${ }^{77}$ IKöln $^{2}$ 397-398. Nr.486 = AE 2010, 1014 = EDCS Nr.47700105 = EDH Nr.067446 (jeweils mit entsprechender Lesung). - Das Fragment befindet sich heute im Römisch-Germanischen Museum in Köln (ohne Inv.-Nr.).

$78 \mathrm{Zu}$ den wenigen denkbaren Namen s. OPEL II 171 (Gridis, Gripo), Delamarre 2007, 106 (Griponus). KAJANTO 1965 und KAKOSCHKE 2007 führen keine entsprechenden Namen.

${ }^{79}$ CIL XIII 7921 (Zülpich/Tolbiacum), 8844 (am Rhein, genauer Fundort unbekannt). Zum Namen s. SOLIN/SALOMIES 1994², 84, KAKOSCHKE 2006, 190. GN 517, KAKOSCHKE 2012, 104. GN 181.

${ }^{80}$ Zum Namen s. KAKOSCHKE 2007, 394-395. CN 1464.
} 
germanien im zweiten und dritten Jahrhundert n. Chr. üblich, wohl im Dativ genannt wurde. ${ }^{81}$

Die von B. und H. GALSTERER erwogene Ergänzung der Buchstabenreste in Zeile 4 zu posuit ist aus inhaltlichen Gründen fraglich. Vor allem aber lässt der vorhandene Platz am Beginn der Zeile eine entsprechende Ergänzung nicht zu. Wahrscheinlich wurde in der letzten vorhandenen Zeile der Name eines Dedikanten genannt. Hinter dem $\mathrm{V}$ ist ferner noch der Rest einer senkrechten Haste zu erkennen, die zu einem I, L, B, M etc. gehört. Ob in Zeile 4/5 die Lesung Q(uintus) Sulp[ici/us --möglich ist, sei dahingestellt.

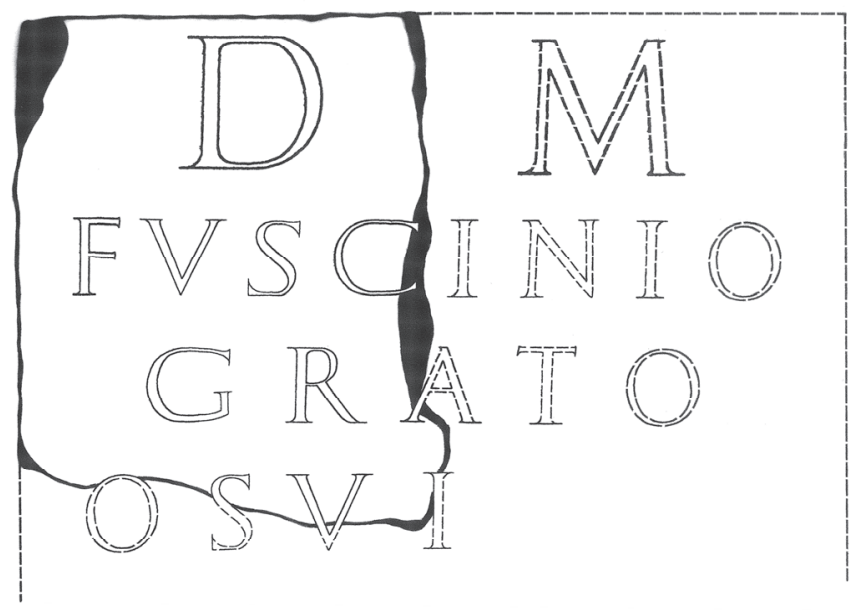

Abb.12: Leicht idealisierte Rekonstruktionszeichnung der fragmentarischen Grabinschrift AE 2010, 1014 aus Köln/CCAA (Zeichnung A.K.)

X.

Die folgende Inschrift stammt ebenfalls aus Köln/CCAA. Der fragmentarische Grabstein (Höhe $89 \mathrm{~cm}$ - Breite 35,5 cm - Tiefe 21,5 cm) fand sich 1883 in der Friesenstraße, zwischen Norbertstraße und Steinfelder Gasse, am Südende des Gräberfeldes um St. Gereon. Bei dem Kalkstein (Abb.13) handelt es sich um die linke Seite einer Giebelstele aus der Zeit zwischen 150 und 250 n. Chr. B. und H. GALSTERER geben den Text unter Bezugnahme auf ältere Lesungen in Umschrift wie folgt wieder: ${ }^{82}$

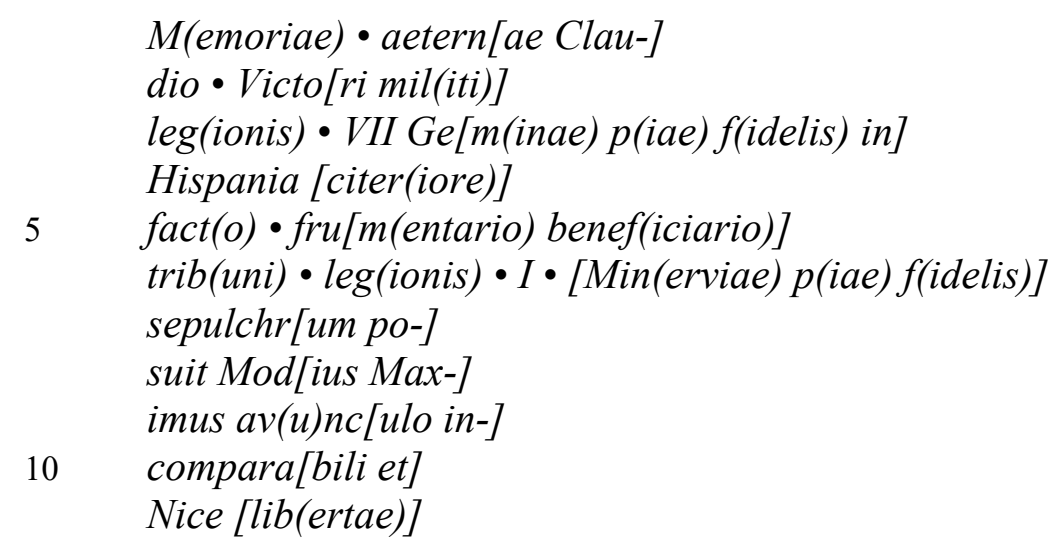

\footnotetext{
81 Zur Verwendung des Dativs (statt des Genitivs) nach $D($ is $)$ M(anibus) in Niedergermanien s. P. KNEIßL, Chiron 5 (1975), 415-416.

${ }^{82}$ IKöln $^{2}$ 266-267. Nr.313. - Der Stein befindet sich heute im Römisch-Germanischen Museum in Köln (Inv.-Nr.139).
} 


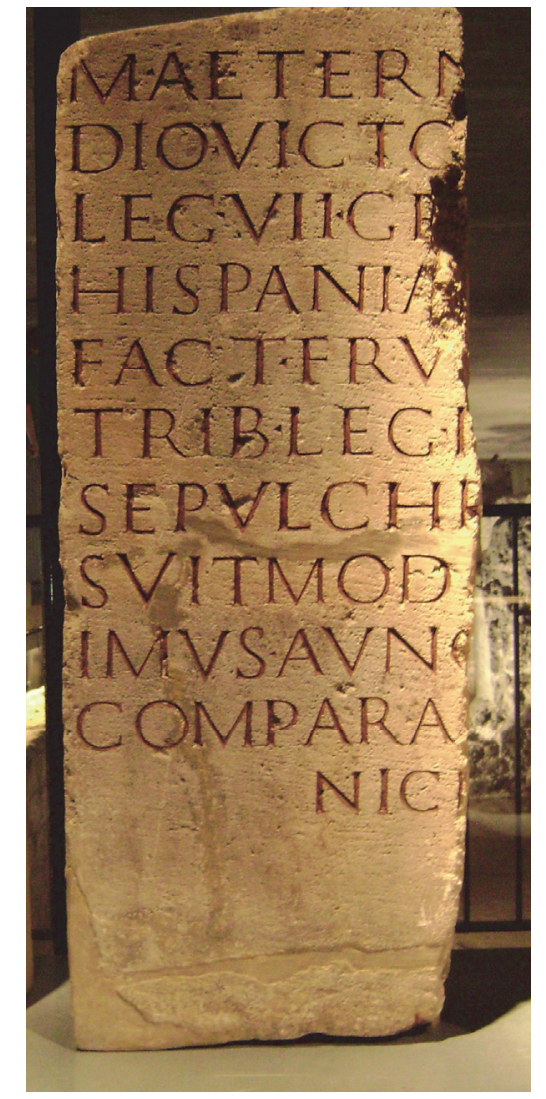

Abb.13: Grabinschrift aus Köln/CCAA: CIL XIII 8282 (C) A.K.)

Eine Rekonstruktionszeichnung (Abb.14) zeigt, dass diese Lesung angesichts der Platzverhältnisse in drei Punkten nicht stimmen kann. Die Größe der Stele lässt sich aufgrund der sicher zu ergänzenden Zeilen 2, 6, 7 und 9 relativ genau bestimmen. Eine zusätzliche Hilfe bietet der noch vorhandene Giebel, dessen höchste Stelle ungefähr über der rechten Hälfte des R in der ersten Zeile liegt. Demzufolge hatte die Stele eine Breite von ca. $60 \mathrm{~cm}$. Am Ende der ersten Zeile kann daher nicht wie bisher angenommen das Gentiliz Claudius gelesen werden. ${ }^{83}$ Zwar lassen die Platzverhältnisse die Lesung aetern[(ae) Clau]/dius zu, doch spricht der unvollständig erhaltene Name des Dedikanten in Zeile 8 eher für eine andere Ergänzung. Der Name des Enkels, der den Stein für seinen Großvater setzte, begann mit MOD. Unter Berücksichtigung der bekannten Gentilnomina und der Tatsache, dass am Ende der Zeile 8 noch der Anfang des Cognomens stand, wurde das Gentiliz seit MOMMSEN ${ }^{84}$ berechtigterweise zu Modius ergänzt. ${ }^{85}$ Da das Gentilnomen des Großvaters mit DIVS

83 Die Ergänzung stammt von TH. MommsEN, WZK 2 (1883), 63. Nr.171. Sie wurde von allen folgenden Autoren und Datenbanken übernommen: s. u.a. DüNTZER 1885, 78-79. Nr.156, J. KLINKENBERG, BJb 108 (1902), 142. Nr.113, CIL XIII 8282 („Zangemeister contulit.“), RIESE 1914, 69. Nr.538, Clauss 1973, 192. Anm.182, GALSTERER 1975, 55. Nr.217. Taf.48 (Photo) [,,Z. 1: Die Ergänzung des Namens ist beliebig; das zunächst naheliegende Modius (Z. 8) ist zu kurz."], P. KNEIßL, Chiron 5 (1975), 415, FAUST 1998, 123. Nr.100, StAUner 2004, 255. Nr.51, LUPA Nr.20705, EDCS Nr.01200120.

${ }^{84}$ TH. Mommsen, WZK 2 (1883), 63. Nr.171.

85 Solin/SAlOMIES 1994², 120-121 verzeichnet noch folgende mit Mod- beginnende Namen: Moderatius, Modestinius, Modestius, Modiarius, Modiasius, Modicius, Modieius, Modinnius, Modioleius. Alle Namen sind zu lang und bis auf Modestius [EDCS Nr.34900227 (Merida/Emerita)] auch nicht in Hispanien belegt. Für Modius finden sich dagegen weitere Nachweise in den hispanischen 
endet, sprechen die Platzverhältnisse in Zeile 1 und der ergänzte Familienname des Dedikanten für die Lesung aetern[ae Mo]/dius. Enkel und Großvater trugen somit höchstwahrscheinlich dasselbe Gentiliz. ${ }^{86}$

In Zeile 5 ist die Ergänzung zu fru[m(entario) benef(iciario)] zu lang. Denkbar wäre fru[m(entario) bene(ficiario)] oder fru[men(tario) b(ene)f(iciario)], doch bleiben auch diese Lesungen fraglich. Schließlich ist das am Ende von Zeile 8 ergänzte MAX zu lang. Vielleicht lautete das Cognomen des Dedikanten eher Primus. ${ }^{87}$ B. und H. GALSTERER schlagen noch Decimus und Ultimus vor. ${ }^{88}$ Die Namen erscheinen aber ebenfalls zu lang.

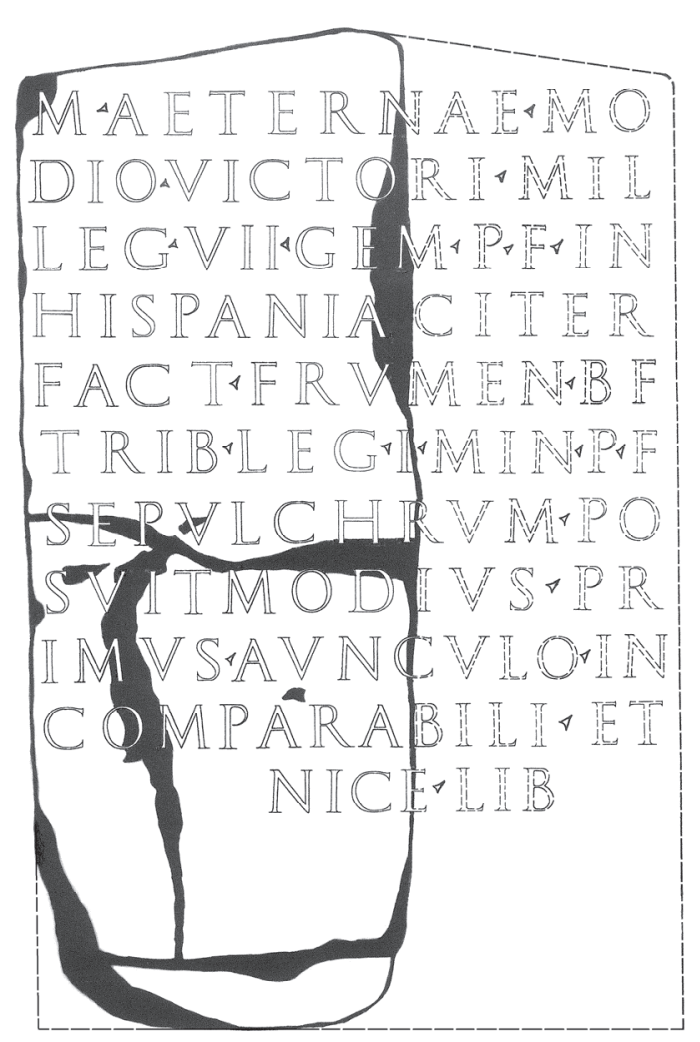

Abb.14: Leicht idealisierte Rekonstruktionszeichnung der fragmentarischen Grabinschrift CIL XIII 8282 aus Köln/CCAA (Zeichnung A.K.)

Zusammenfassend sollte der Text unter Vorbehalten also so gelesen werden:

\footnotetext{
Provinzen [CIL II 1448 (El Hachillo/Olaurum), 5080 (Astorga/Asturica Augusta), II²/7 146, 147 (beide Montoro/Epora)]. - Zu denken gibt noch der relativ große freie Raum nach MOD am Ende der erhaltenen Zeile 8. Eigentlich müsste man im unteren Bereich der Bruchkante noch den Rest eines Buchstabens erkennen können. Dies ist aber nicht der Fall. Offensichtlich hat der Steinmetz nach MOD den Abstand zum folgenden Buchstaben etwas weiter gesetzt. Ein abgekürztes Gentiliz Mod(---) ist sicher auszuschließen, zumal sich nach MOD auch kein Worttrenner erkennen lässt. Möglicherweise fehlte an der Bruchkante mehr Steinmaterial als nach der Restaurierung der Inschrift zu erkennen ist.

${ }^{86}$ Entsprechend führt das OPEL III 85 die Inschrift CIL XIII 8282 mit zwei Nachweisen für das Gentiliz Modius. Zu Modius s. ferner KaKoschKe 2006, 276. GN 812.

${ }^{87}$ [Pr]imus lasen auch schon Th. MOMMSEN, WZK 2 (1883), 63. Nr.171, J. KlinKENBERG, BJb 108 (1902), 142. Nr.113 und RIESE 1914, 69. Nr.538.

${ }^{88}$ GALSTERER 1975, 55. Nr.217. Taf.48 (Photo).
} 


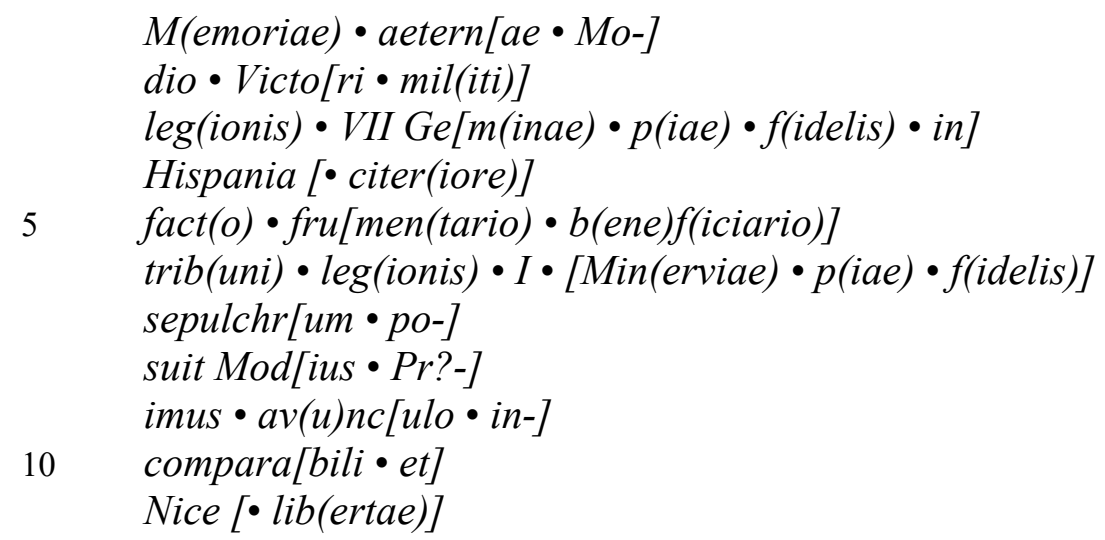

XI.

Im Jahre 1990 publizierte SCHALLMAYER einen in Osterburken (Neckar-OdenwaldKreis) entdeckten fragmentarischen Votivaltar (Abb.15) aus der Zeit zwischen 200 und $250 \mathrm{n}$. Chr. Der Weihestein aus grünlichem Schilfsandstein (Höhe 35,5 cm Breite $39 \mathrm{~cm}$ - Tiefe 28,5 cm) fand sich im bekannten Beneficiarierweihebezirk von Osterburken (Befund XVIII/4, Fund-Nr.1103). Die Inschrift des unvollständigen Altars wurde mit Hilfe von noch zu erkennenden Hilfslinien sorgfältig, wenn auch teilweise etwas gedrängt, eingeschlagen. SCHALLMAYER gab den noch erhaltenen stark abgekürzten Text mit einer Buchstabenhöhe von 5 bis $4,5 \mathrm{~cm}$ in Umschrift wie folgt wieder: ${ }^{89}$

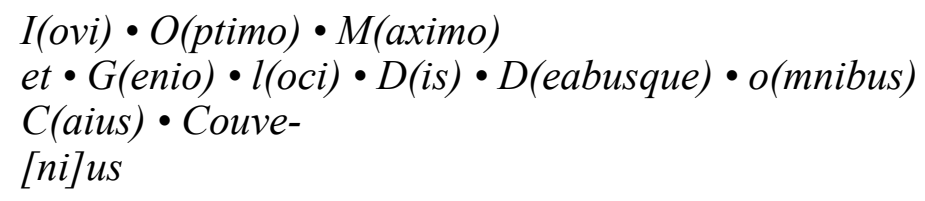

Angesichts des Fundortes vermutete SchallMaYer in dem Dedikanten zu Recht einen Beneficiarier. Man kann also mit ziemlicher Sicherheit davon ausgehen, dass in den ca. zwei bis drei verlorenen Zeilen abgesehen vom Cognomen des Dedikanten zumindest noch die wohl abgekürzte Angabe b(ene)f(iciarius) co(n)s(ularis) und die gängige Schlussformel v(otum) s(olvit) l(ibens) m(erito) (oder ähnlich) standen.

Unter Verweis auf bei HOLDER belegte keltische Wortstämme mit Cou- und $\mathrm{Cov}^{-}{ }^{90}$ sah SCHALLMAYER in dem Gentiliz des Dedikanten überzeugenderweise einen Namen keltischen Ursprungs. Allerdings kann die Lesung Couve/[ni]us nicht zutreffen. Bereits die Wiedergabe der Inschrift in der L'Annee Épigraphique brachte einen Fortschritt. Hier lautete die Lesung des Gentilnomens berechtigterweise Couue/[...]ius, da vor den noch erhaltenen Buchstaben VS eindeutig noch der Rest eines I zu erkennen ist, das leicht über die obere Grundlinie der Zeile hinausragt. Ferner verwies der Kommentar zur Inschrift SCHALLMAYER folgend auf verwandte keltische Namen hin, wie Covilinus (CIL XIII 4412, Metz/Divodurum), Cousius (2. N. 56. Nr.14, Trier/Augusta Treverorum) und Covirius (CIL XIII 11313, 1. N. 8-9. Nr.26, Trier/Augusta Treverorum) ${ }^{91}$ und stellte hinsichtlich des vorliegenden Namens zutreffend fest, dass das Gentiliz Covvenius nicht weiter bezeugt ist und die von

\footnotetext{
${ }^{89}$ Schallmayer 1990, 151. Nr.165. - Der Stein befindet sich heute in der Sammlung des Römermuseums in Osterburken (Inv.-Nr.LDA KA EV 83/3-1103).

${ }^{90}$ HoldER 1896-1907, Bd.1. 1150-1151. S. jetzt auch Delamarre 2007, 76.

${ }^{91} \mathrm{Zu}$ den Namen s. auch KAKOSCHKE 2010, 75-76. GN 148-149, 293. CN 450 (mit weiteren Belegen).
} 
SCHALLMAYER ergänzten Buchstaben die Lücke am Beginn der vierten Zeile nicht ausfüllen. ${ }^{92}$

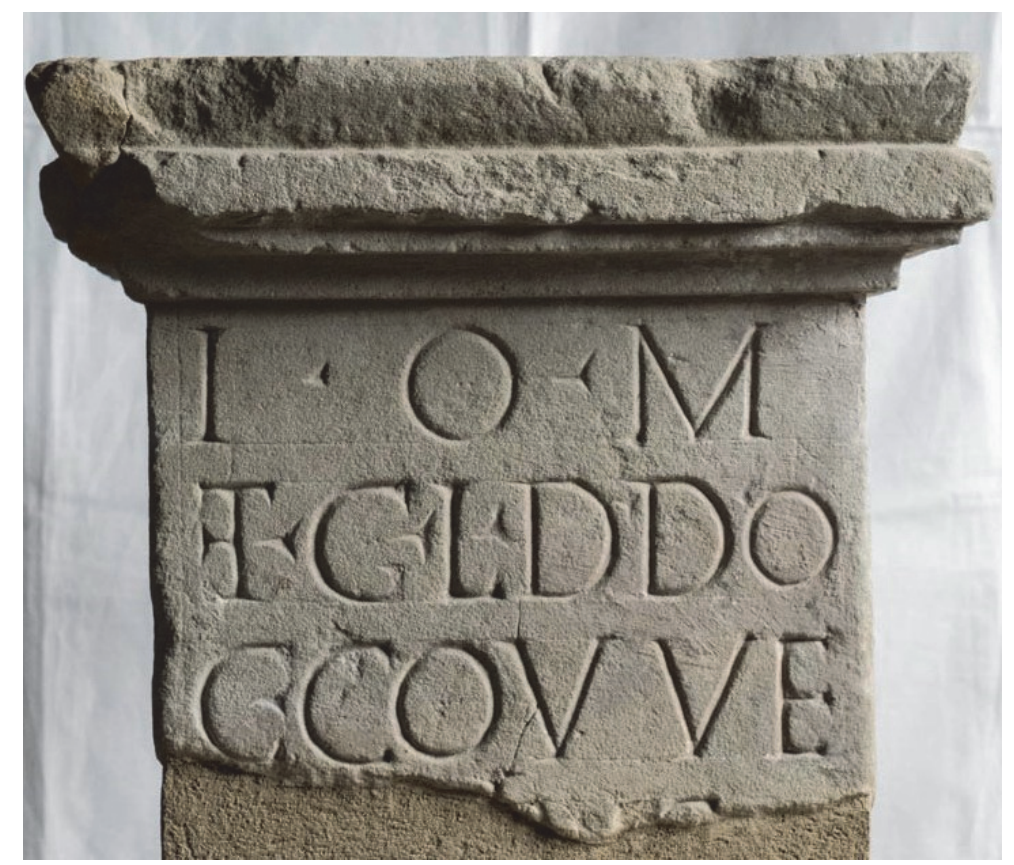

Abb.15: Fragmentarische Weihinschrift aus Osterburken: AE 19961195 (C) Ortolf Harl, Wien)

Auf der Suche nach einer möglichen Ergänzung trifft man in Gundershoffen (dép. Bas-Rhin), im Gebiet der Triboker, auf einen Beleg für das nomen simplex Coventus. ${ }^{93}$ Der Name kommt in der vorliegenden Inschrift aus Osterburken als Basis für das unvollständige Pseudogentiliz jedoch nicht in Frage, da Covve/[nt]ius - hier mit geminiertem V - aus Platzgründen nicht denkbar ist. ${ }^{94}$ Der ergänzte Name füllt die vierte Zeile am Beginn nicht ganz aus. Vergleichbares gilt für den durch einen Töpfer in Rheinzabern/Tabernae bezeugten Namen Covventio, ${ }^{95}$ da Covve/[ntion]ius für den Beginn der vierten Zeile zu lang ist. Dies gilt offensichtlich auch für den Fall, dass die verlorene Buchstabengruppe NTION mit Ligaturen eingeschlagen wurde.

Durchmustert man die bisher bekannten Gentil- und Cognomina aus den zwei germanischen Provinzen und den anliegenden Regionen, trifft man lediglich auf einen Namen, der in der vorliegenden Inschrift ergänzt werden kann: Covventinius. Die Basis für dieses bisher nicht bezeugte Pseudogentiliz, der keltische Name Covventinus, findet sich im nicht allzu weit von Osterburken entfernten Heddernheim/Nida. ${ }^{96}$ Wahrscheinlich gehörte das in Zeile 4 noch zu erkennende lange I zu einer vielfach bezeugten NI-Ligatur. Ferner ist aus Platzgründen wohl mit einer weiteren Ligatur zu rechnen. In der Rekonstruktion (Abb.16) wurde hier eine gängige TI-Ligatur favorisiert.

\footnotetext{
92 AE 1996, 1159. S. ferner EDH Nr.HD36897 und LUPA Nr.25690 mit der Lesung Couve/[n?]ius. In der EDCS Nr.03000694 lautet die Lesung des Namens Cove/[3]ius(!). Solin/SALOMIES 1994², 482 und KaKoschKe 2007, 152. GN 371 führen den Namen als Couve[ni?]us. Vgl. auch noch KaKOSCHKE 2014, 50, 52 (Couve[ni]us, ein hapax legomenon).

${ }^{93}$ CIL XIII 6028 (Iulius Coventi). Zum Namen s. KAKoschKe 2007, 269. CN 943.

${ }^{94}$ Vgl. entsprechende Überlegungen bei KaKOSCHKE 2002, 261. Anm.1335.

${ }^{95}$ HARTLEY/DiCKINSON 2008b, 162-163.

${ }^{96}$ AE 2010, $1087=$ KAKOSCHKE 2002, 260-262. Nr.2.42.
} 
Das Cognomen Covventinus ist offenbar theophoren Ursprungs und leitet sich von der keltischen Göttin Coventina ab. ${ }^{97}$ Die Göttin wurde unter den Namen Coventina, Covetina, Covventina und Covvintina an einer Quelle bei Carrawburgh/Procolitia (Northumberland) am Hadrianswall verehrt. Die Weihungen stammen fast ausschließlich von Germanen, von Stammesmitgliedern der Bataver und Cugernern, deren Einheiten an der britannischen Nordgrenze stationiert waren. ${ }^{98}$ Womöglich bezieht sich auch eine Weihung aus der Hispania citerior an Convetene auf dieselbe Göttin. ${ }^{99}$

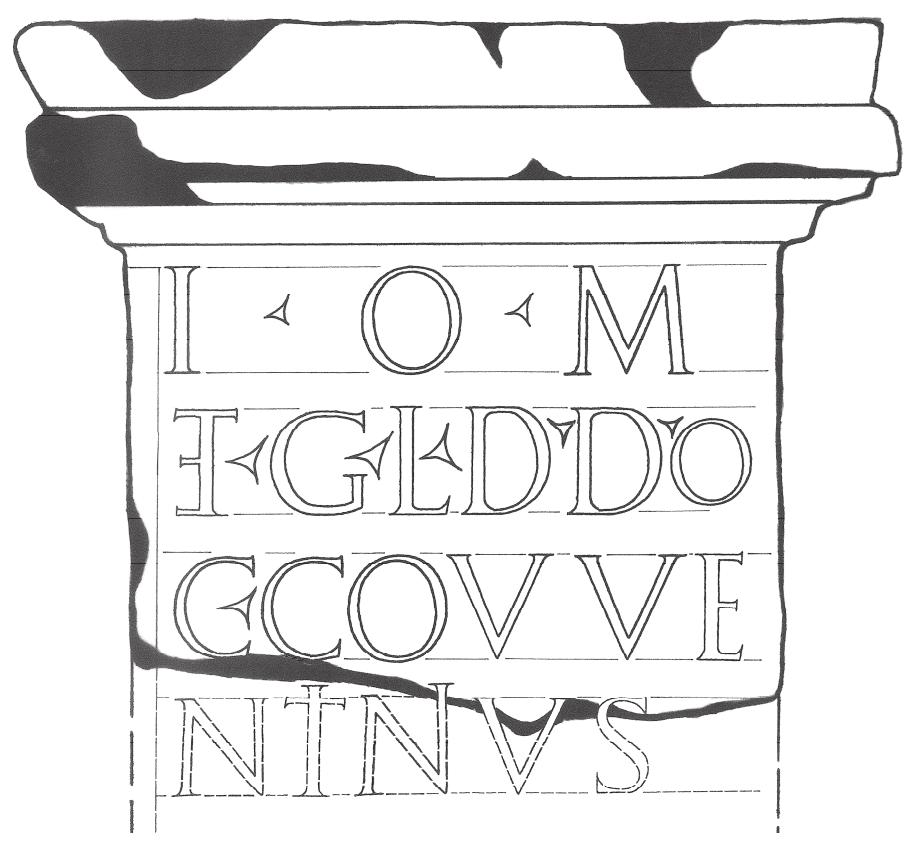

Abb.16: Leicht idealisierte Rekonstruktionszeichnung der fragmentarischen Weihinschrift AE 1996, 1195 aus Osterburken (Zeichnung A.K.)

\section{Siglen}

1. N.

2. N.

3. N.

4. N.
H. FINKE, Neue Inschriften, BRGK 17 (1927), 1-107, 198-231.

H. Nesselhauf, Neue Inschriften aus dem römischen Germanien und den angrenzenden Gebieten, BRGK 27 (1937), 51-134.

H. Nesselhauf / H. Lieb, Dritter Nachtrag zu CIL. XIII. Inschriften aus den germanischen Provinzen und dem Treverergebiet, BRGK 40 (1959), 120-229.

U. SCHILLINGER-HÄFELE, Vierter Nachtrag zu CIL XIII und zweiter Nachtrag zu FR. VoLLMER, Inscriptiones Baivariae Romanae. Inschriften aus dem deutschen An-

\footnotetext{
${ }^{97}$ Zum Namen s. KAKOSCHKE 2007, 269. CN 944.

${ }^{98}$ RIB $^{2}$ 1522-1529, 1531-1535. Zu den Funden s. E. HÜBNER, Hermes 12 (1877), 257-271.

99 AE 1950, $24=1954,251=$ EDH Nr.HD021583 (Parga); s. auch AE 1957, 322. Zur Gottheit s. M. IHM, RE IV.2 (1901), 1687-1688 (s.v. Coventina), HOLDER 1896-1907, Bd.1. 1111-1112, E. BIRLEY, ANRW II.18.1 (1986), 45-46, M. LE GLAY, LIMC III.1 (1986), 306 (s.v. Coventina).
} 
$\mathrm{AE}$

ANRW

$\mathrm{BJb}$

BRGK

CIL

ES

GFA

IKöln ${ }^{2}$

ILTG

LIMC

OPEL I'IV $-\mathrm{IV}$

RE

$\mathrm{RIB}^{2}$

RiNRW

$\mathrm{TZ}$

WZ

WZK teil der germanischen Provinzen und des Treverergebietes sowie Rätiens und Noricums, BRGK 58 (1977), 447-603.

L'Annee Épigraphique.

Aufstieg und Niedergang der römischen Welt. Geschichte und Kultur Roms im Spiegel der neueren Forschung, Berlin-New York.

Bonner Jahrbücher.

Bericht der Römisch-Germanischen Kommission.

Corpus Inscriptionum Latinarum, Berlin.

Epigraphische Studien.

Göttinger Forum für Altertumswissenschaft. (www.gfa.gbv.de)

B. GALSTERER / H. GALSTERER, Die römischen Steininschriften aus Köln. IKöln ${ }^{2}$, Kölner Forschungen 10, Mainz 2010.

P. Wuilleumier, Inscriptions latines des trois Gaules (France), Gallia Supplément 17, Paris 1963.

Lexicon Iconographicum Mythologiae Classicae, Zürich-München.

Onomasticon Provinciarum Europae Latinarum I2-IV. Ex materia ab A. Mócsy, R. FELDMANN, E. MARTON et M. SZILÁGYI collecta, hrsg. von B. LÖRINCZ u.a., Budapest-Wien 1999-2005.

Paulys Real-Enzyklopädie der classischen Altertumswissenschaft, Stuttgart.

R. G. Collingwood / R. P. Wright, The Roman Inscriptions of Britain 1. Addenda and Corrigenda by R. S. O. TOMLIN, Oxford 1995.

H. G. HORN (Hrsg.), Die Römer in Nordrhein-Westfalen, Stuttgart 1987.

Trierer Zeitschrift für Geschichte und Kunst des Trierer Landes und seiner Nachbargebiete.

Westdeutsche Zeitschrift für Kunst und Geschichte.

Westdeutsche Zeitschrift für Kunst und Geschichte / Korrespondenzblatt.

http://www.fera-journal.eu 


\section{Literaturverzeichnis}

BAUCHHENß 2001

BRAMBACH 1867

BURNS 1994

Clauss 1973

DELAMARRE 2007

DÜNTZER 1885

FAUST 1998

GALSTERER 1972/73

GALSTERER 1975

GALSTERER 1981

GUTENBRUNNER 1936

HOLDER 1896-1907
G. BAUCHHENß, Bonnerinnen und Bonner zur Römerzeit - In Inschriften und der antiken Literatur genannte Personen, die sich in Bonn aufgehalten haben, in: M. VAN REY (Hrsg.), Bonn von der Vorgeschichte bis zum Ende der Römerzeit, Bonn, 312-354.

W. BRAMBACH, Corpus Inscriptionum Rhenanarum, Elberfeld.

V. T. BURNS, Romanization and acculturation: The Rhineland matronae, Ann Abor [= Diss. (Mikrofiche) Wisconsin-Madison.

M. Clauss, Untersuchungen $\mathrm{zu}$ den principales des römischen Heeres von Augustus bis Diokletian. Cornicularii, speculatores, frumentarii, Diss. Bochum.

X. Delamarre, Nomina Celtica antiqua selecta inscriptionum (Noms de personnes celtiques dans l'épigraphie classique), Paris.

H. DÜNTZER, Verzeichnis der römischen Alterthümer des Museums Wallraf-Richartz in Köln ${ }^{3}$, Köln.

W. FAUST, Die Grabstelen des 2. und 3. Jahrhunderts im Rheingebiet, Beihefte der Bonner Jahrbücher 52, Bonn.

B. Galsterer / H. Galsterer, Neue Inschriften aus Köln, KJb 13, 92-101.

B. GALSTERER / H. GALSTERER, Die römischen Steininschriften aus Köln, Wissenschaftliche Kataloge des Römisch-Germanischen Museums 2, Köln.

B. Galsterer / H. Galsterer, Neue Inschriften aus Köln - Funde der Jahre 1974-1979, ES 12 (Sammelband), 225-264.

S. GUTENBRUNNER, Die germanischen Götternamen der antiken Inschriften, Rheinische Beiträge und Hülfsbücher zur germanischen Philologie und Volkskunde 24, Halle (Saale).

Hartley/Dickinson 2008a B. R. Hartley / B. M. Dickinson, Names on Terra Sigillata. An Index of Makers' Stamps \& Signatures on Gallo-Roman Terra Sigillata (Samian Ware), Bd.1. A to AXO, London.

Hartley/Dickinson 2008b B. R. Hartley / B. M. Dickinson, Names on Terra Sigillata. An Index of Makers' Stamps \& Signatures on Gallo-Roman Terra Sigillata (Samian Ware), Bd.3. CERTIANUS to EXSOBANO, London.

A. HoldER, Alt-celtischer Sprachschatz. 3 Bde., Leipzig. (Nachdr. Graz 1961-1962.)

http://www.fera-journal.eu 
HÜPSCH 1801

KAJANTO 1965

KAKOSCHKE 2002

KAKOSCHKE 2006

KAKOSCHKE 2007

KAKOSCHKE 2008

KAKOSCHKE 2010

KAKOSCHKE 2012

KAKOSCHKE 2014

KoLBE 1960

LEHNER 1918

NOELKE 1981

RAEPSAET-CHARLIER 2001
A. VON HÜPSCH, Epigrammatographia sive collectio inscriptionum antiquioris medii et recentioris aevi provinciarum Germaniae inferioris. Köln.

I. Kajanto, The Latin Cognomina, Societas Scientiarum Fennica. Commentationes Humanarum Litterarum 36.2, Helsinki-Helsingfors.

A. KAKOschKe, Ortsfremde in den römischen Provinzen Germania inferior und Germania superior. Eine Untersuchung zur Mobilität in den germanischen Provinzen anhand der Inschriften des 1. bis 3. Jahrhunderts n. Chr., Osnabrücker Forschungen zu Altertum und Antike-Rezeption 5, Möhnesee.

A. KaKoschKe, Die Personennamen in den zwei germanischen Provinzen. Ein Katalog. Bd.1: Gentilnomina ABILIUS-VOLUSIUS, Rahden/Westf.

A. KakoschKe, Die Personennamen in den zwei germanischen Provinzen. Ein Katalog. Bd.2,1: Cognomina ABAIUS-LYSIAS, Rahden/Westf.

A. KaKoschKe, Die Personennamen in den zwei germanischen Provinzen. Ein Katalog. Bd.2,2: Cognomina MACCAUS-ZYASCELIS, Rahden/Westf.

A. KakoschKe, Die Personennamen in der römischen Provinz Gallia Belgica, Alpha - Omega. Reihe A. Lexika - Indizes - Konkordanzen zur klassischen Philologie 255, Hildesheim-Zürich-New York.

A. KaKoschKe, Die Personennamen in der römischen Provinz Noricum, Alpha - Omega. Reihe A. Lexika Indizes - Konkordanzen zur klassischen Philologie 263, Hildesheim-Zürich-New York.

A. KAKOSCHKE, Hapax - Steinmetzirrtum - Überlieferungsfehler - Fehllesung - Fälschung. Zu einigen auffälligen Personennamen aus den zwei germanischen Provinzen, Stuttgart.

H.-G. KolBE, Die neuen Matroneninschriften von Morken-Harff, Kr. Bergheim, BJb 160, 50-124.

H. LEHNER, Die antiken Steindenkmäler des Provinzialmuseums in Bonn, Veröffentlichungen des Provinzialmuseums in Bonn 9, Bonn.

P. NOELKE, Die Iupitersäulen und -pfeiler in der römischen Provinz Germania inferior, Beihefte der Bonner Jahrbücher 41, Köln-Bonn.

M.-TH. RAEPSAET-CHARLIER, Onomastique et romanisation: élémentes d'une comparaison entre les provinces de Gaule Belgique et de Germania inférieure, in:

http://www.fera-journal.eu 
RIESE 1914

SCHALLMAYER 1990

SCHOPPA 1959

SCHULZE 1904

SOLIN/SALOMIES $1994^{2}$

SPAUL 2000

STAUNER 2004

STOLLWERCK 1877

THOMAS 2004

WEISGERBER 1968
M. DONDIN-PAYRe / M.-TH. RAEPSAET-CHARLIER (Hrsg.), Noms, identités culturelles et romanisation sous le Haut-Empire, Bruxelles, 399-472.

A. RIESE, Das rheinische Germanien in den antiken Inschriften, Leipzig-Berlin.

E. Schallmayer u.a., Der römische Weihebezirk von Osterburken I. Corpus der griechischen und lateinischen Beneficiarier-Inschriften des Römischen Reiches, Forschungen und Berichte zur Vor- und Frühgeschichte in Baden-Württemberg 40, Stuttgart.

H. SchорPA, Römische Götterdenkmäler in Köln, Die Denkmäler des römischen Köln 22, Köln.

W. SCHUlZE, Zur Geschichte lateinischer Eigennamen, Abhdlg. der Königl. Ges. der Wiss. zu Göttingen. Phil.Hist. K1. N. F. 5, Göttingen (Nachdr. Berlin-ZürichDublin 1966).

H. Solin / O. SAlomies, Repertorium nominum gentilium et cognominum Latinorum. 2. Aufl. Editio nova addendis corrigendisque augmentata, Alpha Omega. Reihe A. Lexika - Indizes - Konkordanzen zur klassischen Philologie 80, Hildesheim-Zürich-New York.

J. E. H. Spaul, Cohors ${ }^{2}$. The evidence for and a short history of the auxiliary infantry units of the Imperial Roman Army, BAR International Series 841, Oxford

K. StaunER, Das offizielle Schriftwesen des römischen Heeres von Augustus bis Gallienus (27 v. Chr. - 268 n. Chr.). Eine Untersuchung zu Struktur, Funktion und Bedeutung der offiziellen militärischen Verwaltungsdokumentation und zu deren Schreibern, Bonn.

F. STOLLWERCK, Die celtubisch-römische Niederlassung Gelduba zwischen Novesium und Asciburgium, Uerdingen.

R. ThOmAS, Denkmäler der Matronenverehrung in der CCAA (Köln), KJb 47, 91-178.

J. L. WeISGERBER, Die Namen der Ubier, Wissenschaftliche Abhandlungen der Arbeitsgemeinschaft für Forschung des Landes Nordrhein-Westfalen 34, KölnOpladen. 
Frankfurter elektronische Rundschau zur Altertumskunde 32 (2017)

\section{Elektronische Datenbanken}

EDCS

Epigraphik-Datenbank Clauss/Slaby (Frankfurt), Leitung: M. CLAUSS (Johann Wolfgang Goethe-Universität Frankfurt am Main), Stand: 17.01.2017 (www.manfredclauss.de).

$\mathrm{EDH}$

Epigraphische Datenbank Heidelberg, Leitung: CH. WITSCHEL (Ruprecht-Karls-Universität Heidelberg), Stand: 17.01.2017 (www.uni-heidelberg.de/institute/ sonst/adw/edh).

LUPA

UBI ERAT LUPA, Bilddatenbank zu antiken Steindenkmälern (Universität Salzburg), Leitung: F. und O. HARL (Universität Wien), Stand: 17.01.2017 (www.ubi-eratlupa.org).

\section{Kontakt zum Autor:}

Dr. Andreas Kakoschke

Nelly-Sachs-Weg 1

D-49191 Belm

E-Mail: andreaskakoschke@hotmail.de 